
\title{
Besearch S Surare \\ Trajectory Tracking Control of Mobile Manipulators Subject to Unknown External Forces
}

Miroslaw Galicki ( $\square$ M.Galicki@ibem.uz.zgora.pl)

Faculty of Mechanical Engieering https://orcid.org/0000-0001-7934-8058

\section{Research Article}

Keywords: mobile manipulator , unstructured external forces , trajectory tracking , robust finite-time task space control , Lyapunov stability

Posted Date: July 21st, 2021

DOl: https://doi.org/10.21203/rs.3.rs-715787/v1

License: (9) This work is licensed under a Creative Commons Attribution 4.0 International License. Read Full License 


\title{
Trajectory tracking control of mobile manipulators subject to unknown external forces
}

\author{
Mirosław Galicki
}

Received: date / Accepted: date

\begin{abstract}
This work proposes a new class of controllers for mobile manipulators subject to both undesirable forces exerted on the end-effector and slip reaction forces acting on the platform wheels, unknown friction forces coming from joints directly driven by the actuators as well as undesirable forces caused by kinematic singular configurations appearing on the mechanism trajectory. Based on suitably defined task space non-singular terminal sliding manifold (TSM) and the Lyapunov stability theory, we derive a class of estimated extended transposed Jacobian controllers which seem to be effective in counteracting the unstructured forces. Moreover, in order to eliminate (or to alleviate) undesirable chattering effects, the proposed control law involves second order sliding technique. The numerical computations closely related to an experiment, which are carried out for a mobile manipulator consisting of a platform of $(2,0)$ type and holonomic manipulator of two revolute kinematic pairs, illustrate the performance of the proposed controllers.
\end{abstract}

Keywords mobile manipulator · unstructured external forces · trajectory tracking · robust finite-time task space control · Lyapunov stability

\section{Introduction}

In the recent years, an interest has increased in applying the mobile manipulators to high-tolerance assembly tasks such as precise welding or cutting, peg-in-hole assembly, material handling, etc. Nevertheless, accomplishing the tasks requiring extremely high accuracy still makes a great challenge for mobile

M. Galicki

Faculty of Mechanical Engineering, University of Zielona Góra, Zielona Góra, Poland.

Tel.: +48-68-3282614

Fax: +48-68-3282614

E-mail: m.galicki@iim.uz.zgora.pl 
manipulators due to various uncertainties of payloads (e.g. parts to be assembled, end-effector tools, etc.), which are rigidly gripped and transferred by the end-effector along a desired trajectory mostly expressed in task (Cartesian) coordinates. In practice, for example, either a known or unknown payload (by its geometry and mass) introduces structural and/or parametric uncertainties in both kinematic and dynamic equations of the mobile manipulator. As a result, transferred payloads generate undesirable external forces acting on the end-effector, which may result in large tracking errors. Similarly, obstacles generating artificial repulsive forces which act on the end-effector may lead to significant degradation of the trajectory tracking. Moreover, movement of the end-effector on a surface of an obstacle generates undesirable friction caused by force contact. Another important source of uncertainty is related to the slipping of the platform wheels when tracking by the end-effector the desired trajectory. This undesirable feature fundamentally distinguishes stationary manipulators from the mobile ones and makes the control of the latter extremely difficult. As is known, the ideal no-slipping and pure rolling (nonholonomic) constraints are mostly violated when the mobile platform either accelerates or decelerates or turns at a high speed. Alternatively, the wheels may also slip in the case of acting the external forces on the end-effector (e.g. transferring of heavy payload or the tool at the end-effector is applying force on the workpiece). If this is the case, slip reaction forces are induced, which disturb the platform motion and, as a consequence, generate the errors in tracking the end-effector trajectory. The relation between slip reaction forces and the wheels slips is very hard to analyse because of such unstructured factors as tire deformation, tire adhesion, tire wear and tear, wheel-surface (ground) conditions, tire pressure, load, etc. Although in most situations met in practice, the end-effector tracking tasks are expressed in Cartesian coordinates, a wide majority of commercially available controllers are designed in the generalized (joint) coordinates. Consequently, inverse or pseudo-inverse kinematics algorithms are first required to apply the aforementioned controllers. The process of kinematic inversion is both time consuming and becomes very complicated when the Cartesian trajectory generates kinematic and/or algorithmic singularities [7]. Consequently, in order to extend the potential applications of the mobile manipulators to tasks requiring the high accuracy of the accomplishment, it is necessary to control the end-effector position by taking into account the interaction forces (undesirable forces from e.g. payloads exerted on the end-effector and/or slip reaction forces acting on the platform wheels, etc.). Moreover, reaction forces caused by (isolated) singular configurations appearing when tracking the desired trajectory by the end-effector must also be taken into account.

\subsection{Related works}

In the context of motion control problem subject to external forces coming e.g. from unstructured payload carried by the end-effector, a few approaches my 
be distinguished, among which two ones seem to be the most representative: trajectory tracking and force regulation as well as trajectory tracking and external force compensation. The vast majority of the motion/force control algorithms is designed in the generalized (joint) coordinates (see e.g. [1]-[6]). Unfortunately, these controllers can not be directly applicable to tracking the desired end-effector trajectory specified in task (Cartesian) coordinates. A few works have been devoted to solving the problem of both trajectory tracking and force compensation in the task space [8]-[10]. Nevertheless, the control laws proposed in [8]-[10] require both the full knowledge of the kinematic and dynamic equations. Moreover, controllers from [8]-[10] need both generalized pseudo-inverse of the Jacobian matrix and external force measurements. The approach discussed in the work [11] is based on the application of the (generalized) pseudo-inverse of the mobile manipulator Jacobian matrix in the control formulation. Nevertheless, steering algorithm from [11] requires full knowledge of the kinematic equations. As is known, the radius of the wheels (e.g. wheels with rubber tires) of the mobile platform is most often not exactly known. Using both fuzzy logic system and a robust term, the trajectory tracking controller proposed in work [12] generates discontinuous steering signals. Furthermore, the controller from [12] requires full knowledge of the kinematic equations. In the work [13], a robust acceleration control scheme based on the disturbance observer designed in joint space has been proposed to accomplish a desired motion in the Cartesian space under conditions of acting the external forces on the end-effector. However, the control law from [13] needs both force measurement and the full knowledge of the kinematic equations of the mechanism as well as a pseudo-inverse of the Jacobian matrix. Recent works [14]-[16] propose adaptive control schemes to estimate and compensate an unknown external force exerted on the end-effector tracking a desired trajectory. The control laws in [14]-[16] involve all the adaptive terms multiplied by the regression matrix which seems to be both complex to implement and time consuming. Moreover, control algorithms from [14]-[17] are not able to tackle singular configurations. Furthermore, the controllers proposed in [14][17] require full knowledge of the kinematic equations and the work [17] needs additionally force sensor. The vast majority of works on slips of the wheels is devoted only to mobile platforms (mobile robots). Works [18]-[19] incorporate the wheel slips into dynamic model of mobile robot. The authors from [18][19] assumed linear dependence of slip reaction forces on small slip rations and then developed a slow manifold approach to design output feed-back control law based on the full knowledge of kinematics and dynamics of the mobile platform. A position controller based on feed-back linearisation has been presented in [20] using the so-called multicycle approach to a four wheeled mobile robot. The algorithms from [20] require measurements of the robot acceleration and velocity to estimate the lateral and longitudinal slips as well as full knowledge of the kinematic and dynamic models. In works [21]-[22], lateral and longitudinal slip variables are included into the overall dynamics of the robot. The authors from [21]-[22] have developed a position control law to asymptotically stabilize the tracking error to zero provided that the reaction forces are not sat- 
urated. Nevertheless, control algorithms from [21]-[22] require full knowledge of the kinematic and dynamic equations as well as lateral and longitudinal slip variables, which have to be measured using techniques reported in e.g. [23]. A robust tracking control scheme has been proposed in [24] for wheeled mobile robots in the presence of wheel slips, which is based on the adaptive disturbance observer estimating the slip variables. The controller from [24] needs also full knowledge of kinematics and dynamics of the mobile robot. Only a very few works has been reported which tackle the problem of slips in the mobile manipulators. In [25], a concept of active control is introduced to avoid wheel slip caused by unstructured road conditions. By properly changing the configuration of the holonomic manipulator in [25], the vertical forces acting on the wheels (which are close to slipping) have larger values. However, the knowledge of the wheel driving torques is required in control algorithm from [25]. Moreover, tracking the trajectory using the algorithm from [25] seems to be problematic. In study [26], a method is developed to determine the slip condition of a wheeled mobile manipulator. Slip analysis presented in [26] is concerned with the reaction wrench from the holonomic manipulator onto the mobile platform.

\subsection{Motivation of the work}

In this paper, a new class of controllers for mobile manipulators subject to both undesirable forces exerted on the end-effector and slip reaction forces acting on the platform wheels, undesirable forces resulting from singular configurations appearing on the mechanism trajectory as well as unknown friction forces coming from joints directly driven by the actuators, is introduced. Due to unstructured nature of the external disturbance forces, the kinematics and dynamics of the mechanism is assumed herein to be uncertain. In order to tackle the trajectory tracking control problem subject to the aforementioned unstructured forces, a new non-singular terminal sliding manifold (TSM) is introduced. As is known [27]-[28], sliding mode is accurate and insensitive to disturbances and (parametric, structural) uncertainties of mechanism dynamics. However, the main drawback of the standard first order sliding modes is mostly related to the undesirable chattering effect [29]. The proposed TSM manifold makes it possible to simultaneously join the first order sliding mode approach possessing the finite-time control capabilities with the second order sliding mode techniques generating the (absolutely) continuous controls. Based on the TSM introduced and motivated in part by dynamically computed torque techniques (see our work [31]), we propose a new robust controller (incorporating a transposed extended estimated Jacobian matrix), which tackles both unknown external forces and does not need slip variables. By fulfilment of a reasonable assumption regarding the Jacobian matrix, the proposed control scheme is shown to be finite time stable. As opposed to other task space motion/force controllers (see e.g. [8]-[17]) incorporating either inverse or pseudoinverse of the Jacobian matrix, additional advantage of our transpose extended 
estimated Jacobian approach is that it does not suffer from singularity problem. It is also worth to mention that trajectory tracking controller analysed in our work [31] utilizes a transpose Jacobian matrix. However, it differs significantly from that proposed herein. Namely, controller from [31] requires both full knowledge of the kinematic equations and partial knowledge (actuator matrix) of the mechanism dynamics. Furthermore, external end-effector and slip reaction forces acting on the mobile manipulator are not analysed in [31]. The remainder of the paper is organized as follows. Section 2. introduces kinematic and dynamic equations of the mechanism including also external forces acting on the mobile manipulator. Section 3 sets up a class of robust controllers solving the trajectory tracking problem in a finite-time. Section 4 presents computer examples of the end-effector trajectory tracking by a mobile manipulator, which is subjected to external disturbance forces. Finally, some concluding remarks are drawn in section 5 .

\section{Problem formulation}

2.1 Kinematic and dynamic equations of the mobile manipulator

Let us consider a mobile manipulator composed of a platform of $(2,0)$ type whose non-holonomic constraints are not satisfied. Let $x=\left(x_{1}, \ldots, x_{l}\right)^{T} \in \mathbb{R}^{l}$ $(l \geq 2)$ denote the vector of generalized coordinates of the mobile platform. We shall assume that $k(k<l)$ non-holonomic velocity constraints (possibly overdetermined) $A^{\prime}(x) \dot{x}=0$, where $A^{\prime}(x)$ denotes the $(k \times l)$ singular matrix of the $\operatorname{rank}\left(A^{\prime}(x)\right)=k-1$, given in the Pfaffian form and imposed on the platform wheels are violated. The condition of violation of the non-holonomic constraints can be analytically expressed as follows

$$
A^{\prime}(x) \dot{x}=\dot{\chi}
$$

where $\dot{\chi}=\left(\dot{\chi}_{1}, \ldots, \dot{\chi}_{k}\right)^{T} \in \mathbb{R}^{k}$ denotes vector of wheel lateral and longitudinal slip velocities; $\|\dot{\chi}\|>0$. The configuration of holonomic manipulator attached to the moving platform is defined by the vector of joint (generalized) coordinates $y=\left(y_{1}, \ldots, y_{n}\right)^{T} \in \mathbb{R}^{n}$, where $n$ is the number of kinematic pairs. In particular, Fig. 1 presents an exemplary mobile manipulator whose platform posture is described by variables $x_{1, c}, x_{2, c}, \theta$ and angles of driving wheels $\phi_{1}$, $\phi_{2}$, where $\theta$ is the orientation angle of the platform with respect to global coordinate system $O X_{1} X_{2} X_{3}$; here $x_{1, c}$ and $x_{2, c}$ stand for the coordinates of the mas centre of the platform; $2 W$ denotes the distance between the platform wheels and $2 L$ is its length; $y_{1}, y_{2}$ stand for joint angles of the holonomic manipulator; $d$ is the distance between the mass centre and common drive wheels axis; $a$ stands for the distance between mass centre and the point, at which the holonomic manipulator is rigidly attached to the platform; $R$ denotes the radius of the wheel; $F$ is an external force exerted on the end-effector and $\chi_{1}, \chi_{2}$ as well as $\chi_{3}, \chi_{4}$ denote lateral and longitudinal slip variables of the 


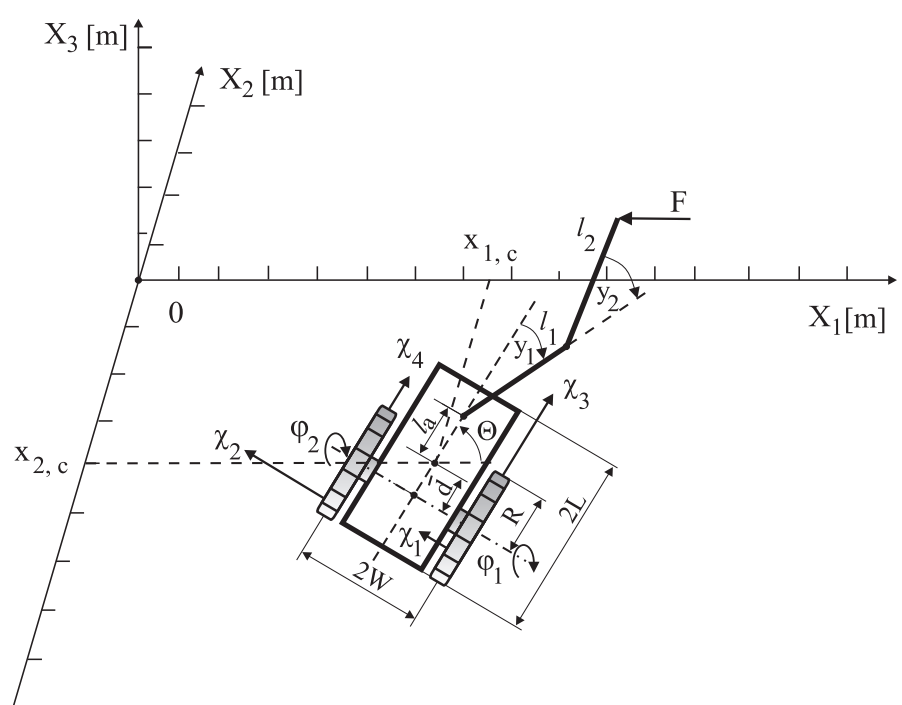

Fig. 1 A kinematic scheme of the mobile manipulator with both external forces $F$ acting on the end-effector and slip variables $\chi_{1}, \ldots, \chi_{4}$.

platform wheels, respectively. Equation (1) may be equivalently rewritten as

$$
A(\chi, x) \frac{d}{d t}\left(\begin{array}{c}
\chi \\
x
\end{array}\right)=0
$$

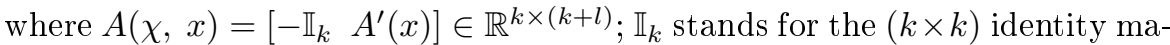
trix. Let us note that matrix $A(\chi, x)$ has full rank equal to $\operatorname{rank}(A)=k$. Thus, equality (2) may also be interpreted as non-holonomic constraints of a Pfaffian form expressed in extended generalized platform coordinates $\left(\begin{array}{l}\chi \\ x\end{array}\right)$. Let $\operatorname{Ker}(A(\chi, x))$ be a null space generated by vector fields $a_{1}(\chi, x), \ldots, a_{l}(\chi, x)$, respectively. Hence, differential constraints (2) may be equivalently expressed as a drift-less dynamic system of the form

$$
\frac{d}{d t}\left(\begin{array}{l}
\chi \\
x
\end{array}\right)=N(\chi, x) \alpha,
$$

where $N(\chi, x)=\left[a_{1}(\chi, x), \ldots, a_{l}(\chi, x)\right] \in \mathbb{R}^{(k+l) \times l} ; \operatorname{rank}(N(\chi, x))=l$ and $\alpha=\left(\alpha_{1}, \ldots, \alpha_{l}\right)^{T}$ may be interpreted as vector of quasi-velocities (introduced in work [34]). Let us note that

$$
A(\chi, x) N(\chi, x)=0 .
$$

Location and orientation of the end-effector with respect to the global coordinate system $O X_{1} X_{2} X_{3}$ is described by the kinematic equation

$$
p_{e}=f_{e}(q),
$$


where $p_{e} \in \mathbb{R}^{m}$ denotes the coordinates of the end-effector; $q=\left(\begin{array}{l}\chi \\ x \\ y\end{array}\right)$ is extended configuration of the mobile manipulator; $f_{e}: \mathbb{R}^{k} \times \mathbb{R}^{l} \times \mathbb{R}^{n} \longrightarrow \mathbb{R}^{m}$ represents $m$-dimensional mapping (in general, non-linear with respect to $q$ ) and $m$ is the dimension of the task (Cartesian) space. Combining $\dot{q}, \ddot{q}$ and (3), one obtains the following expressions:

$$
\dot{q}=C z, \quad \ddot{q}=C \dot{z}+\dot{C} z,
$$

where $C(\chi, x)=\left[\begin{array}{cc}N(\chi, x) & 0 \\ 0 & \mathbb{I}_{n}\end{array}\right] \in \mathbb{R}^{(k+l+n) \times(l+n)} ; z=\left(\begin{array}{c}\alpha \\ \dot{y}\end{array}\right) \in \mathbb{R}^{l+n}$ is reduced mobile manipulator velocity; $\mathbb{I}_{n}$ denotes the $(n \times n)$ identity matrix. On account of the fact that mobile manipulator becomes mostly in practice a redundant mechanism with respect to a task to be accomplished, the following inequality holds true $l+n-k+1 \geq m$. Consequently, there exists a possibility to augment vector of the end-effector coordinates, describing the classic (conventional) trajectory tracking, by additional task coordinates (specified by the user) of the following general form [35]:

$$
p_{a}=f_{a}(q)
$$

where $f_{a}: \mathbb{R}^{k+l+n} \longrightarrow \mathbb{R}^{l+n-m-k+1}$ is at least triply continuously differentiable mapping with respect to $q$. From the practical point of view, redundant degrees of freedom of the mechanism may either satisfy additional task requirements (constraints) [30], [35] or optimize performance criteria reflecting the kinematic characteristics of the mobile manipulator [36]. Concatenating $f_{e}(q)$ with $f_{a}(q)$, one obtains generalized kinematic-differential mappings which relate $q$ with augmented task coordinates $p=\left(\begin{array}{c}p_{e} \\ p_{a}\end{array}\right)$

$$
p=f(q), \quad \dot{p}=\jmath(q) z,
$$

where $f=\left(\begin{array}{l}f_{e} \\ f_{a}\end{array}\right)$ and $\jmath=\frac{\partial f}{\partial q} C$ is the $(l+n-k+1) \times(l+n)$ extended Jacobian matrix. Applying the Lagrange formalism [4] to the mobile manipulator described by extended configuration $q$, introducing vectors of friction forces coming from joints directly driven by the actuators, external forces exerted on the end-effector and slip reaction forces acting on the platform wheels as well as taking into account differential constraints (2), we can arrive at the following system of dynamic equations:

$$
M^{\prime}(q) \ddot{q}+P^{\prime}(q, \dot{q}) \dot{q}+G^{\prime}(q)+\left[A(\chi, x) 0_{k \times n}\right]^{T} \lambda=B^{\prime} K_{r} K_{t} v+E^{\prime}(q, \dot{q}),
$$

where $M^{\prime}(q)$ denotes the $(k+n+l) \times(k+n+l)$ positive definite inertia matrix; $P^{\prime}(q, \dot{q}) \dot{q}$ is the $(k+n+l)$-dimensional vector representing centrifugal and Coriolis forces; $\dot{q}$ denotes the mobile manipulator extended velocity; $G^{\prime}(q)$ stands for the $(k+n+l)$-dimensional vector of generalized gravity forces; 
$E^{\prime}(q, \dot{q})=E_{f}^{\prime}(\dot{q})+E_{e}^{\prime}(q)+E_{s}^{\prime}(\dot{q})+E_{g}^{\prime}\left(\cup\left\{q^{\prime}\right\}\right)$ describes friction, external endeffector and slip reaction generalized forces as well as reaction force vector coming from kinematic constraints (5) for (isolated) singular configurations $q^{\prime}$, respectively; $0_{k \times n}$ denotes the $k \times n$ zero matrix; $\lambda$ is the $k$-dimensional vector of Lagrange multipliers corresponding to differential constraints (2); $B^{\prime}=\left[\begin{array}{rr}B^{\prime \prime} & 0 \\ 0 & \mathbb{I}_{n}\end{array}\right] ; B^{\prime \prime}$ stands for the $(k+l) \times(l-k+1)$ matrix indicating which configuration variables of the platform are directly driven by the actuators (its elements equal 1 for configuration variables directly driven by the actuators and 0 otherwise); $K_{r}, K_{t}$ are the $(l+n-k+1) \times(l+n-k+1)$ diagonal transmission and torque constants matrices, respectively; $v \in \mathbb{R}^{n+l-k+1}$ is the vector of controls (armature currents). The friction forces acting in the mobile manipulator joints directly driven by the actuators take the following simplified form:

$$
E_{f}^{\prime}(\dot{q})=\left(E_{f, 1}^{\prime}, \ldots, E_{f, k+l+n}^{\prime}\right)^{T},
$$

where $E_{f, i}^{\prime}=-\gamma_{i} \dot{q}_{i}$ for $q_{i}$ directly driven by the $i$-th actuator and $E_{f, i}^{\prime}=0$ otherwise; $\gamma_{i}>0$ denotes the viscous friction coefficient; $i=1, \ldots, k+l+n$. The external end-effector forces expressed in the extended generalized coordinates are given as follows

$$
E_{e}^{\prime}(q)=D^{\prime}(q) F,
$$

where $D^{\prime}(q)$ represents the $(k+l+n) \times m$ matrix which transforms external $m$-dimensional forces $F$ expressed in task coordinates and exerted on the endeffector into corresponding generalized forces acting in mechanism joints. Let us observe, that e.g. equations of motion of the payload $o$ rigidly gripped by the end-effector are as follows $M_{o}\left(p_{e}\right) \ddot{p}_{e}=-F-C_{o}\left(p_{e}, \dot{p}_{e}\right) \dot{p}_{e}-G_{o}\left(p_{e}\right)$, where $M_{o}\left(p_{e}\right), C_{o}\left(p_{e}, \dot{p}_{e}\right) \dot{p}_{e}$ and $G_{o}\left(p_{e}\right)$ denote (known or unknown/unstructured) inertia matrix, centrifugal and generalized gravity forces, respectively of the payload $o ; o \in \mathcal{O} ; \mathcal{O}$ stands for a set of all admissible payloads to be rigidly gripped and transferred by the end-effector. The presence of the slip induces at the contact points of the platform wheels with the ground reaction forces $E_{s}^{\prime}$ counteracting the slips. Since slips have the sense of velocities, this means that the slip reaction forces directly depend on $\dot{q}$. The value of lateral slip reaction force for the $j$-th wheel of the mobile platform from Fig. 1 depends on slip angle $s a_{j}=\tan ^{-1}\left(\frac{\dot{\chi}_{j}}{\left|R \dot{\phi}_{j}-\dot{\chi}_{j}\right|}\right)$, where $j=1,2$. Moreover, the longitudinal slip reaction force depends on slip ratio $s r_{j}=\frac{\dot{\chi}_{j}}{\max \left\{\left|R \dot{\phi}_{j}\right|,\left|R \dot{\phi}_{j}-\dot{\chi}_{j}\right|\right\}}$, where $j=1,2$. Using variables $s r_{j}$ and $s a_{j}$, we can model both lateral and longitudinal slip reaction forces based on Pacejka or Magic formula [48], which has been widely accepted for use in industry and academia. The general form of slip reaction forces with Pacejka formula is given below

$$
E_{s}^{\prime}(\dot{q})=\left(E_{s, 1}^{\prime}, \ldots, E_{s, k+l+n}^{\prime}\right)^{T},
$$

where $E_{s, j}^{\prime}=K_{1, j} \sin \left(K_{2, j} \tan ^{-1}\left(K_{3, j} s a_{j}+K_{4, j}\left(\tan ^{-1}\left(K_{3, j} s a_{j}\right)-K_{3, j} s a_{j}\right)\right)\right)+$ $K_{5, j} ; E_{s, j+2}^{\prime}=K_{1, j}^{\prime} \sin \left(K_{2, j}^{\prime} \tan ^{-1}\left(K_{3, j}^{\prime} s r_{j}+K_{4, j}^{\prime}\left(\tan ^{-1}\left(K_{3, j}^{\prime} s r_{j}\right)-K_{3, j}^{\prime} s r_{j}\right)\right)\right)+$ 
$K_{5, j}^{\prime}$ for $j=1,2$ and $E_{s, j}^{\prime}=0$ otherwise; $K_{1, j}, K_{1, j}^{\prime}, \ldots, K_{5, j}, K_{5, j}^{\prime}$ are constant parameters determined from the hyper-surface fitting process of the empirical data related to the tire and ground characteristics. An alternative continuously differentiable model of the slip reaction forces has been proposed in work [23]. For relatively small values of variables $s a_{j}$ and $s r_{j}$, the Pacejka formula may be linearised. Thus, we obtain simplified expressions of slip reaction forces $E_{s, j}^{\prime}$ in the following form: $E_{s, j}^{\prime}=-C_{j} s a_{j}$ for lateral slip reaction force and $E_{s, j+2}^{\prime}=-D_{j} s r_{j}$ for longitudinal slip reaction force, respectively; $C_{j}, D_{j}$ denote cornering stiffness coefficient and slip stiffness coefficient, respectively depending on the nature of the wheels and the ground. In order to avoid numerical problems that may appear for small values of $\left|R \dot{\phi}_{j}-\dot{\chi}_{j}\right|$, a saturation technique may be introduced. In particular, if $\left|R \dot{\phi}_{j}-\dot{\chi}_{j}\right|<\delta$ then $\left|R \dot{\phi}_{j}-\dot{\chi}_{j}\right|$ is replaced by $\delta$, where $\delta$ is a small positive number. Moreover, if $\max \left\{\left|R \dot{\phi}_{j}\right|,\left|R \dot{\phi}_{j}-\dot{\chi}_{j}\right|\right\}<\delta$ then $\max \left\{\left|R \dot{\phi}_{j}\right|,\left|R \dot{\phi}_{j}-\dot{\chi}_{j}\right|\right\}$ is replaced by $\delta$, too. Otherwise, if $s a_{j}$ and $s r_{j}$ take relatively large values then models for the slip reaction forces offered in [23], [48] should be used. Reaction generalized force $E_{g}^{\prime}\left(\cup\left\{q^{\prime}\right\}\right)$ takes the form

$$
E_{g}^{\prime}=\sum_{\left\{q^{\prime}: \operatorname{rank}\left(D^{\prime}\left(q^{\prime}\right)<m\right)\right\}} D^{\prime}\left(q^{\prime}\right) F_{g}\left(q^{\prime}\right),
$$

where $F_{g}\left(q^{\prime}\right)$ is reaction force vector coming from kinematic constraints (5) for (isolated) singular configuration $q^{\prime}$. Let us note that, due to singular matrix $D^{\prime}\left(q^{\prime}\right)$, it seems very hard to obtain an analytical form for $F_{g}\left(q^{\prime}\right)$. Replacing $\dot{q}$ and $\ddot{q}$ from (9) by reduced velocity $z$ and acceleration $\dot{z}$ (see expressions (6)), we significantly simplify our control problem. Premultiplying left-sided the dynamic equations (9) by $C^{T}$ and then using the equality $C^{T}\left[A(\chi, x) 0_{k \times n}\right]^{T}=0$ (see expression (4)), we obtain dynamic equations of the mobile manipulator in the following reduced form, which is convenient for our control purposes:

$$
M(q) \dot{z}+P(q, z) z+G(q)=B(q) v+E(t, q, z),
$$

where $M=C^{T} M^{\prime} C \in \mathbb{R}^{(l+n) \times(l+n)}$ denotes positive definite symmetric reduced inertia matrix; $P=C^{T}\left(M^{\prime} \dot{C}+P^{\prime} C\right) ; G=C^{T} G^{\prime} ; E=C^{T} E^{\prime} ; B=$ $C^{T} B^{\prime} K_{r} K_{t}$. Let us observe that dynamics (14) is characterized by deficit of controls variables with respect to the number of coordinates of vector $z$ $(l+n>l+n-k+1$ for $k>1)$.

\subsection{The trajectory tracking control problem to be solved}

The task accomplished by the mobile manipulator is to track both desired end-effector trajectory $p_{d}^{e}(t) \in \mathbb{R}^{m}, t \in[0, \infty)$ and auxiliary (user specified) trajectory $p_{d}^{a}(t) \in \mathbb{R}^{l+n-m-k+1}$. Vector functions $p_{d}^{e}(\cdot)$ and $p_{d}^{a}(\cdot)$ are assumed to be at least triply continuously differentiable with respect to time. Introducing the task tracking error $e=\left(\begin{array}{c}e^{e} \\ e^{a}\end{array}\right)=f(q)-p_{d}(t)$, where $p_{d}=\left(\begin{array}{c}p_{d}^{e} \\ p_{d}^{a}\end{array}\right)$; 
$e^{e}=\left(e_{1}^{e}, \ldots, e_{m}^{e}\right)^{T}=f_{e}-p_{d}^{e} ; e^{a}=\left(e_{1}^{a}, \ldots, e_{l+n-m-k+1}^{a}\right)^{T}=f_{a}-p_{d}^{a}$, the finite time control problem may be formally expressed by means of the following equations:

$$
\lim _{t \rightarrow T} e(t)=0, \lim _{t \rightarrow T} \dot{e}(t)=0, \lim _{t \rightarrow T} \ddot{e}(t)=0,
$$

where $0 \leq T$ denotes a finite time of convergence of $f(q)$ to $p_{d}$ and $e(t)=\dot{e}(t)=$ $\ddot{e}(t)=0$ for $t \geq T$. Due to requirement $f_{e}(q)-p_{d}^{e}(t)=0$ in (15), external force $F$ exerted by the payload $o$ on the end-effector takes the form $F=$ $-M_{o}\left(p_{d}^{e}(t)\right) \ddot{p}_{d}^{e}(t)-C_{o}\left(p_{d}^{e}(t), \dot{p}_{d}^{e}(t)\right) \dot{p}_{d}^{e}(t)-G_{o}\left(p_{d}^{e}(t)\right), o \in \mathcal{O}$. Let us note that, it is practically impossible to precisely define set $\mathcal{O}$ which implies uncertainty of force $F$. In what follows, we introduce useful generalized extended Jacobian matrix $J(q) \in \mathbb{R}^{(l+n-k+1) \times(l+n-k+1)}$ of the form

$$
J(q)=\jmath(q) M^{-1}(q) B(q)
$$

Moreover, we introduce practically reasonable assumptions and summarize useful properties regarding both kinematic and dynamic equations which will be utilized while designing our controller. Without loss of generality of further considerations, matrix $J$ and $\jmath$ together with its derivatives up to the second order with respect to $q$ are assumed to be bounded, i.e.

$$
\|J\|_{F},\left\|\frac{\partial \jmath}{\partial q}\right\|_{F},\left\|\frac{\partial^{2} \jmath}{\partial q^{2}}\right\|_{F}<\infty,
$$

where $\|\cdot\|_{F}$ stands for the Frobenius (Euclidean) matrix norm. The components of dynamic equations (9) fulfil the following inequalities for revolute kinematic pairs of the holonomic manipulator [4]:

$$
\begin{aligned}
& \|C\|_{F},\left\|\frac{\partial C}{\partial q}\right\|_{F},\left\|M^{\prime}\right\|_{F},\left\|\frac{\partial M^{\prime}}{\partial q}\right\|_{F}, \\
& \left\|\frac{\partial P^{\prime}}{\partial \dot{q}}\right\|,\left\|\frac{\partial G^{\prime}}{\partial q}\right\|_{F}<\infty
\end{aligned}
$$

and

$$
\left\|\frac{\partial P^{\prime}}{\partial q}\right\|_{F} \leq w_{P^{\prime}}\|z\|,
$$

where $w_{P^{\prime}}$ denotes a positive coefficient. Generalized external force vector $E$ together with its time derivative $\dot{E}$ are assumed in (14) to be at least locally bounded Lebesgue measurable mappings. Without loss of generality, $E$ and $\dot{E}$ are upper estimated as follows

$$
\|E\| \leq \beta^{0}(t, q, z), \quad\|\dot{E}\| \leq \beta^{1}(t, q, z),
$$

where $\beta^{0}(\cdot), \beta^{1}(\cdot)$ stand for the time-dependent non-negative locally bounded Lebesgue measurable functions. However, mappings $\beta^{0}(\cdot)$ and $\beta^{1}(\cdot)$ are allowed to be globally unbounded with respect to their variables. 


\section{Control of the mobile manipulator in the augmented task space}

Before we propose the control law solving the kinematic task (15), some useful concepts are first introduced. Let $\hat{J}=\hat{J}(q)$ denote an estimate of the uncertain generalized extended Jacobian matrix $J(q)$ given by formula (16).

3.1 Dynamic controller of the mobile manipulator

In further considerations, $\hat{J}$ is assumed to fulfil the following inequalities:

$$
0<A \leq \lambda_{\min }\left(\hat{J} \hat{J}^{T}\right)
$$

and

$$
0 \leq\|J-\hat{J}\|_{F} \leq \rho,
$$

where $\rho=\left\{\begin{array}{lr}\rho^{\prime} & \text { for }\|\hat{J}\|_{F} \leq 1 \\ \rho^{\prime} /\|\hat{J}\|_{F} & \text { otherwise; }\end{array} ; \rho\right.$ denotes an upper bound on the accuracy of estimation; $\rho^{\prime} \in[0, A)$. It is worth to note that inequality (22) can be in practice fulfilled by selection of e.g. nominal values of kinematic and dynamic parameters, which are usually given with sufficient accuracy. Although inequality (22) requires sufficiently accurate estimate $\hat{J}$ of Jacobian matrix $J(q)$, the advantage of fulfilment of inequality $(22)$ by $\hat{J}$ is the elimination of the parameter adaptation process. If inequality (22) does not hold true then $\hat{J}$ has to be computed based on the adaptation of kinematic and dynamic as well as drive train (elements of matrices $K_{r}, K_{t}$ ) parameters of the mobile manipulator. This issue will be discussed in the next section (see Remark 3.). Let us also observe that inequality (21) means non-singularity of estimated Jacobian matrix $\hat{J}(q)$ in an operation region of the end-effector. Nevertheless, relations (21)-(22) are only needed in the proof of the finite-time stability of the controller to be designed. However, in what follows, we shall also allow (isolated) singular configurations appearing when trajectory tracking, which certainly do not fulfil inequality (21). In order to obtain at least absolutely continuous control $v$ solving the robotic task (15), let us differentiate dynamic equations (14) with respect to time and then determine $\ddot{z}$ (let us observe that $M(q)$ is non-singular)

$$
\ddot{z}=M^{-1} B \dot{v}-\mathcal{R},
$$

where $\mathcal{R}=M^{-1}\left[\dot{M} \dot{z}+\frac{d}{d t}(P z+G)-\frac{d}{d t} E\right]$. In order to reduce task errors $e$, $\dot{e}$ and $\ddot{e}$ in a finite time to zero subject to generalized external forces $E$, we propose a new dynamic control law of the form

$$
\dot{v}=\hat{J}^{T} u
$$

where $u \in \mathbb{R}^{l+n-k+1}$ denotes a new control to be determined. The objective is to find input signal $u(t)$ and consequently control $v(t)$ such that extended 
location vector $p$ from (8) follows $p_{d}$. For this purpose, let us triply differentiate $e$ with respect to time thus obtaining

$$
\dddot{e}=\jmath \ddot{z}+2 j \dot{z}+\ddot{j} z-\dddot{p}_{d} .
$$

Replacing $\ddot{z}$ from (25) by the right-hand sides of (23) and (24), one obtains an explicit form of the task jerk error $\dddot{e}$ from $u$

$$
\dddot{e}=J \hat{J}^{T} u+\mathcal{Q}-\dddot{p}_{d},
$$

where $\mathcal{Q}=-\jmath \mathcal{R}+2 j M^{-1}(v+E-P z-G)+\ddot{\jmath} z$. In the sequel, we shall need an upper estimate of $\|\mathcal{Q}\|$. Using inequalities (17)-(20) and performing simple but time consuming algebraic computations, we find upper estimate for $\|\mathcal{Q}\|$ of the form given below

$$
\|\mathcal{Q}\| \leq \mathcal{W}
$$

where $\mathcal{W}=w_{1}\|z\|+w_{2}\|z\|^{3}+w_{3}\|z\| \beta^{0}+w_{4} \beta^{1}+w_{5}\left\|z\left|\|\mid\| v \| ; w_{1}, \ldots, w_{5}\right.\right.$ are positive constants (estimates of construction parameters of the mobile manipulator). Let $s=\left(s_{1}, \ldots, s_{l+n-k+1}\right)^{T} \in \mathbb{R}^{l+n-k+1}$ be a task space sliding vector variable. In order to find steering signal $u$, we propose the following new non-singular terminal sliding manifold $\mathcal{S}$ :

$$
\mathcal{S}=\{(s \ddot{e}(0) \text { e } \dot{e} \ddot{e}): \mathcal{M}(s, \ddot{e}(0), e, \dot{e}, \ddot{e})=0\},
$$

where $\mathcal{M}=s-\ddot{e}+\ddot{e}(0)-\int_{0}^{t}\left(\lambda_{2} \ddot{e}^{3 / 5}+\lambda_{2} \lambda_{1}^{3 / 5}\left(\dot{e}^{9 / 7}+\lambda_{0}^{9 / 7} e\right)^{1 / 3}\right) d \tau ; \lambda_{i}=$ $\operatorname{diag}\left(\lambda_{i, 1}, \ldots, \lambda_{i, l+n-k+1}\right) ; \lambda_{i, j}$ denote positive coefficients (controller gains); $i=0: 2 ; j=1: l+n-k+1$. The potency of both $e, \dot{e}, \ddot{e}$ and $\lambda_{0}, \lambda_{1}, \lambda_{2}$ is defined component-wise. It is worth to notice the fact that, unlike the terminal sliding manifolds (TSM) defined in our earlier works [31], [43], relation (28) has a nice and useful property for the initial time moment $t=0$ of control, i.e. $s(0)=0(\operatorname{TSM} s=0$ is attained at $t=0)$. In the sequel, we present a useful lemma [44].

Lemma 1 If $s(t)=0$ for $t \geq T^{\prime}$, where $0 \leq T^{\prime} \leq T$ then task tracking errors $(e, \dot{e}, \ddot{e})$ stably converge in a finite-time to the origin $(e, \dot{e}, \ddot{e})=(0,0,0)$.

In order to satisfy equality constraints (15), the following control law is proposed:

$$
u(t,\|z\|, \dot{e}, \ddot{e})= \begin{cases}-\frac{c}{A} \frac{s}{\|s\|}\left(\mathcal{Y}+c_{0}\right) & \text { for } s \neq 0 \\ 0 & \text { otherwise }\end{cases}
$$

where $\mathcal{Y}=\left\|\lambda_{2} \ddot{e}^{3 / 5}+\lambda_{2} \lambda_{1}^{3 / 5}\left(\dot{e}^{9 / 7}+\lambda_{0}^{9 / 7} e\right)^{1 / 3}-\dddot{p}_{d}\right\|+\mathcal{W} ; c, c_{0}$ are controller gains to be specified further on. Based on (24) and (29), we can determine $v$ (in the Filippov sense [37]) from solution of the following differential equation:

$$
\dot{v}=\hat{J}^{T} u(t,\|z\|, \dot{e}, \ddot{e}) .
$$

Our aim is to give conditions on controller gains $\lambda_{0}, \lambda_{1}, \lambda_{2}, c$ and $c_{0}$, which guarantee fulfilment of equalities (15). Applying the Lyapunov stability theory, we offer the following result. 
Theorem 1 If matrix $\hat{J}$ fulfils inequalities (21)-(22), $\lambda_{0}, \lambda_{1}, \lambda_{2}, c_{0}>0$, $c=\frac{c^{\prime}}{1-\rho^{\prime} / A}, c^{\prime}>1$ and $q, z, e, \dot{e}$ and $\ddot{e}$ are available then control scheme (29)-(30) results in a finite-time stable convergence of task errors $(e, \dot{e}, \ddot{e})$ to the origin $(e, \dot{e}, \ddot{e})=(0,0,0)$.

Proof Consider the following Lyapunov function candidate:

$$
V=\frac{1}{2}\langle s, s\rangle \text {. }
$$

Differentiating (31) with respect to time and taking into account equality $\mathcal{M}=0$ in (28) results in the following expression:

$$
\dot{V}=\left\langle s, \dddot{e}+\lambda_{2} \ddot{e}^{3 / 5}+\lambda_{2} \lambda_{1}^{3 / 5}\left(\dot{e}^{9 / 7}+\lambda_{0}^{9 / 7} e\right)^{1 / 3}\right\rangle .
$$

Based on (24) and (26), one obtains

$$
\dot{V}=\left\langle s, J \hat{J}^{T} u\right\rangle+\left\langle s, \mathcal{Q}+\lambda_{2} \ddot{e}^{3 / 5}+\lambda_{2} \lambda_{1}^{3 / 5}\left(\dot{e}^{9 / 7}+\lambda_{0}^{9 / 7} e\right)^{1 / 3}-\dddot{p}_{d}\right\rangle .
$$

Let us reformulate expression (33) in equivalent and more convenient form for further analysis

$$
\begin{aligned}
\dot{V}= & \left\langle s, \hat{J} \hat{J}^{T} u\right\rangle+\left\langle s,(J-\hat{J}) \hat{J}^{T} u\right\rangle \\
& +\left\langle s, \mathcal{Q}+\lambda_{2} \ddot{e}^{3 / 5}+\lambda_{2} \lambda_{1}^{3 / 5}\left(\dot{e}^{9 / 7}+\lambda_{0}^{9 / 7} e\right)^{1 / 3}-\dddot{p}_{d}\right\rangle .
\end{aligned}
$$

Let us upper estimate the sum of the first two terms of (34). For this purpose, we insert the right-hand side of (29) into (34) thus obtaining

$$
\begin{aligned}
\dot{V}= & -\left\langle s, \hat{J} \hat{J}^{T} \frac{c}{A} \frac{s}{\|s\|}\left(\mathcal{Y}+c_{0}\right)\right\rangle-\left\langle s,(J-\hat{J}) \hat{J}^{T} \frac{c}{A} \frac{s}{\|s\| !}\left(\mathcal{Y}+c_{0}\right)\right\rangle \\
& +\left\langle s, \mathcal{Q}+\lambda_{2} \ddot{e}^{3 / 5}+\lambda_{2} \lambda_{1}^{3 / 5}\left(\dot{e}^{9 / 7}+\lambda_{0}^{9 / 7} e\right)^{1 / 3}-\dddot{p}_{d}\right\rangle .
\end{aligned}
$$

On account of inequalities (21)-(22), we get

$$
\begin{aligned}
\dot{V} \leq & -\|s\| c\left(\mathcal{Y}+c_{0}\right)+\|s\| \frac{\rho^{\prime} c}{A}\left(\mathcal{Y}+c_{0}\right)+ \\
& \left\langle s, \mathcal{Q}+\lambda_{2} \ddot{e}^{3 / 5}+\lambda_{2} \lambda_{1}^{3 / 5}\left(\dot{e}^{9 / 7}+\lambda_{0}^{9 / 7} e\right)^{1 / 3}-\dddot{p}_{d}\right\rangle .
\end{aligned}
$$

Next, we estimate the last term of $\dot{V}$ in (34). Based on definitions of $\mathcal{Q}, \mathcal{Y}$ and inequality (27), we have

$$
\begin{aligned}
\dot{V} \leq & -\|s\| c\left(\mathcal{Y}+c_{0}\right)+\|s\| \frac{\rho^{\prime} c}{A}\left(\mathcal{Y}+c_{0}\right)+\|s\| \mathcal{Y} \leq \\
& -\|s\| c\left(\mathcal{Y}+c_{0}\right)+\|s\| \frac{\rho^{\prime} c}{A}\left(\mathcal{Y}+c_{0}\right)+\|s\|\left(\mathcal{Y}+c_{0}\right) .
\end{aligned}
$$

Consequently, based on the assumptions $c=\frac{c^{\prime}}{1-\rho^{\prime} / A}, c^{\prime}>1$ and $c_{0}>0$ from Theorem 1, one easily obtains that

$$
\begin{aligned}
\dot{V} \leq & -\|s\| c_{0}\left(c-\frac{\rho^{\prime} c}{A}-1\right)-\|s\| \mathcal{Y}\left(c-\frac{\rho^{\prime} c}{A}-1\right)= \\
& -\|s\| c_{0}\left(c^{\prime}-1\right)-\|s\| \mathcal{Y}\left(c^{\prime}-1\right) \leq-\|s\| c_{0}\left(c^{\prime}-1\right) .
\end{aligned}
$$

Since $c_{0}\left(c^{\prime}-1\right)>0$, inequality $(38)$ proves that $s=0$ is stably attainable in a finite time less or equal to $\frac{\sqrt{2 V(0)}}{\left(c^{\prime}-1\right) c_{0}}$. Consequently, from Lemma 1 , it follows that the origin $(e, \dot{e}, \ddot{e})=(0,0,0)$ is stably attainable in a finite time $T$. 
A few remarks may be made regarding the control law (29)-(30) and Theorem 1.

- Remark 1. First, let us observe that controller (29)-(30) does not need force/torque sensors to detect external forces. Nevertheless, most of the works [32]-[33] use these sensors to determine the values of external forces. As is known, force/torque sensors are expensive, they introduce measurement noise and are complicated to implement. Second, the adaptive estimation of both the external forces acting on the end-effector and parameters of dynamic equations is not also required by our controller. On the other hand, recent studies [14]-[15] estimate external forces and need regressor matrix whose construction is not an unambiguous process nor a trivial task in practice. Moreover, it is worth to note, that controller (29)-(30) does not need reduced vector velocity $z$ but scalar non-negative variable $\|z\|$, which may be replaced by its upper estimate. Vector of quasi-velocities $\alpha$ includes some coordinates of both velocities $\dot{x}_{k_{i}} \in\left\{\dot{x}_{1}, \ldots, \dot{x}_{l}\right\}$, which are directly driven by the actuators and slip velocities $\dot{\chi}_{m_{j}} \in\left\{\dot{\chi}_{1}, \ldots, \dot{\chi}_{k}\right\}$, where $1 \leq i \leq a ; a<l ; 1 \leq j \leq l-a ; 1 \leq k_{i} \leq l$ and $1 \leq m_{j} \leq k$. Hence, $z=\left(\begin{array}{l}\dot{\chi}_{s} \\ \dot{x}_{a} \\ \dot{y}\end{array}\right)$, where $\dot{\chi}_{s}=\left(\dot{\chi}_{m_{1}}, \ldots, \dot{\chi}_{m_{l-a}}\right)^{T}$ and $\dot{x}_{a}=\left(\dot{x}_{k_{1}}, \ldots, \dot{x}_{k_{a}}\right)^{T}$. From (1), one obtains $\left\|\dot{\chi}_{s}\right\| \leq c_{A^{\prime}}\|\dot{x}\|$, where $c_{A^{\prime}}$ is a positive coefficient. Let $z_{n}(\dot{x}, \dot{y})$ denote an upper estimate of $\|z\|$. From definition of $z$ and the last inequality, we have

$$
\|z\| \leq z_{n}(\dot{x}, \dot{y})
$$

where $z_{n}(\dot{x}, \dot{y})=c_{A^{\prime}}\|\dot{x}\|+\left\|\left(\begin{array}{c}\dot{x}_{a} \\ \dot{y}\end{array}\right)\right\|$. Consequently, replacing the norm $\|z\|$ in expression (29) by scalar variable $z_{n}(\dot{x}, \dot{y})$, we conclude that control law (29)-(30) does not require the knowledge of slip velocities $\dot{\chi}_{s}$ nor their estimates, which are hard to directly sense. Nevertheless, most of the works use either directly slip variables [21]-[22] or their estimates [24] in the trajectory tracking control what requires the full knowledge of mobile platform kinematic parameters.

- Remark 2. In the particular case $\rho^{\prime}=0$, extended generalized Jacobian matrix $J$ fulfils equality $\hat{J}=J$ (kinematic equations are fully known). Let $J(\cdot)$ be singular at (isolated) configuration $q^{\prime}$ and $0 \neq s \notin \operatorname{ker}\left(J^{T}\left(q^{\prime}\right)\right)$. In such a case, equality (34) from the proof of Theorem 1 takes the following simplified form: $\dot{V}=\left\langle J^{T} s, J^{T} u\right\rangle+\left\langle s, \mathcal{Q}+\lambda_{2} \ddot{e}^{3 / 5}+\lambda_{2} \lambda_{1}^{3 / 5}\left(\dot{e}^{9 / 7}+\lambda_{0}^{9 / 7} e\right)^{1 / 3}-\right.$ $\left.\dddot{p}_{d}\right\rangle$. For control law

$$
u(t,\|z\|, \dot{e}, \ddot{e})= \begin{cases}-c_{s}^{\prime} \frac{s}{\|s\|}\left(\mathcal{Y}+c_{0}\right) & \text { for } s \neq 0 \\ 0 & \text { otherwise }\end{cases}
$$

and

$$
\dot{v}=J^{T} u(t,\|z\|, \dot{e}, \ddot{e}),
$$


where $c_{s}^{\prime}>1$ is a gain coefficient, we have $\dot{V} \leq-\left\|J^{T} s\right\|^{2} \frac{\mathcal{Y}+c_{0}}{\|s\|} c_{s}^{\prime}+\|s\| \mathcal{Y}$. Hence, for sufficiently large $c_{s}^{\prime}, \dot{V}$ takes negative values. Consequently, control law (40)-(41) enables the mobile manipulator with known kinematics and uncertain dynamics to pass also through singular manifold $\left\{q^{\prime}: \operatorname{det}\left(J\left(q^{\prime}\right)\right)=0\right\}$ as the numerical computations carried out in the next section show. It is also worth to mention that control algorithms offered in the literature (see e.g. works [8]-[17]) can not directly tackle singular configurations.

- Remark 3. If inequality (22) is not satisfied then computationally involved parametric adaptation procedure has to be started. For this purpose, let us introduce auxiliary Jacobian matrix $J_{a}(q, p)=\jmath \operatorname{adj}(M) B$, where $\operatorname{adj}(M)$ is the adjoint matrix of $M ; p$ denotes vector of uncertain parameters, which are combinations of physical kinematic and dynamic as well as drive train parameters of the mobile platform and manipulator links. Let $\hat{p}$ be an estimate of unknown parameter vector $p$. Based on definition of Jacobian matrix $J_{a}$, we can propose the following robust adaptive control law provided that inequality (22) is not satisfied: $\dot{v}_{a}=\hat{J}_{a}^{T} u_{a}$, where $\hat{J}_{a}=J_{a}(q, \hat{p}) ; u_{a}$ denotes auxiliary control whose structure is similar to that given by formula (29). Vector $\hat{p}$ is computed based on adaptation law given in differential form, which incorporates sliding vector variable $s$, Jacobian matrix $J_{a}(q, \hat{p})$ and auxiliary steering signal $u_{a}$. Due to the need to find $\hat{p}$, computational complexity of determination of $v_{a}$ is much greater than that resulted from expressions (29)-(30). Finally, steering signals $v_{a}$ provide, in general, only asymptotic stability due to adaptation process of parameter vector $\hat{p}$. Nevertheless, control law $v_{a}$ does not require fulfilment of inequality (22) by vector $\hat{p}$.

- Remark 4. Let us observe that expressions (29)-(30) present a transpose Jacobian controller. In such a context, the use of the transpose of the Jacobian matrix to robotic manipulators in [38]-[40] is a well-known technique. However, works [38]-[40] present stability analysis for the set-point control problems. On the other hand, Theorem 1 provides stability analysis for the trajectory tracking of the mechanisms whose both kinematic and dynamic equations are uncertain as well as disturbances acting on the mobile manipulators are unknown. In such a context, it is worth to note the fact that authors from works [41]-[42] have also shown finite-time convergence of their controller using however the inverse of the Jacobian matrix.

\subsection{The state estimation problem}

Taking into account Remark 1., let us observe that controllers (29)-(30) or (40)-(41) require the knowledge of task error $e$, task velocity error $\dot{e}$, task acceleration error $\ddot{e}$, manipulator joint configuration $y$ and velocity $\dot{y}$ as well as mobile platform posture $x$ and velocity $\dot{x}$, respectively to generate suitable steering signals. In most cases, real mobile manipulators are equipped in joint encoders, which make it possible to measure joint angles $y=y(t)$. Moreover, 
wheel encoders, laser scanners and gyroscope sensors provide measurements of both task error $e=e(t)$ and platform posture $x=x(t)$. There exist many approaches in the literature to reconstruct quantities $\dot{e}, \ddot{e}, \dot{y}$ and $\dot{x}$, respectively (see, for example, our work [43], in which different kinds of state observers were analysed). Almost all of the observers known from the literature have to satisfy the so-called separation principle [45]. Due to discontinuity of control laws (29)-(30), (40)-(41), separation principle is not fulfilled for our controllers. Consequently, an approach based on the theoretical results presented in [46], [47] and also successfully adapted for non-holonomic mechanisms without slips (see our work [31]) will be now proposed for reconstruction of $\dot{e}, \ddot{e}, \dot{y}$ and $\dot{x}$. Let us note that observer from [31] requires the knowledge of kinematic parameters of the mobile platform and additionally reconstruction of $\ddot{y}$ and $\ddot{x}$, respectively. On account of the fact that $e=e(t), y=y(t)$ and $x=$ $x(t)$, are measurable, one can exactly reconstruct $\dot{e}(t), \ddot{e}(t), \dot{y}(t)$ and $\dot{x}(t)$ (by neglecting a measurement noise of the devices) after finite-times of transient processes, say $T_{e}^{\prime}, T_{y}^{\prime}, T_{x}^{\prime}>0$, respectively. The second order uniform robust exact differentiator (model-free observer) for the reconstruction of $\dot{e}, \ddot{e}$ and the first order uniform robust differentiators reconstructing $\dot{y}$ and $\dot{x}$, respectively, take in our case the following forms:

$$
\begin{aligned}
& \dot{\tau}_{0}=\tau_{1}-\hat{\lambda}_{2}^{e} L_{e}(t)^{1 / 3}\left\|\tau_{0}-e\right\|^{2 / 3} \operatorname{sign}\left(\tau_{0}-e\right), \\
& \dot{\tau}_{1}=\tau_{2}-\hat{\lambda}_{1}^{e} L_{e}(t)^{2 / 3}\left\|\tau_{0}-e\right\| \|^{1 / 3} \operatorname{sign}\left(\tau_{0}-e\right), \\
& \dot{\tau}_{2}=-\hat{\lambda}_{0}^{e} L_{e}(t) \operatorname{sign}\left(\tau_{0}-e\right), \\
& \dot{\omega}_{0}=\omega_{1}-\hat{\lambda}_{1}^{y} L_{y}(t)^{1 / 2}\left|\omega_{0}-y\right|^{1 / 2} \operatorname{sign}\left(\omega_{0}-y\right), \\
& \dot{\omega}_{1}=-\hat{\lambda}_{0}^{y} L_{y}(t) \operatorname{sign}\left(\omega_{0}-y\right)
\end{aligned}
$$

and

$$
\begin{aligned}
& \dot{\psi}_{0}=\psi_{1}-\hat{\lambda}_{1}^{x} L_{x}(t)^{1 / 2}\left|\psi_{0}-x\right|^{1 / 2} \operatorname{sign}\left(\psi_{0}-x\right), \\
& \dot{\psi}_{1}=-\hat{\lambda}_{0}^{x} L_{x}(t) \operatorname{sign}\left(\psi_{0}-x\right),
\end{aligned}
$$

where $\hat{\lambda}_{0}^{e}, \hat{\lambda}_{1}^{e}, \hat{\lambda}_{2}^{e}, \hat{\lambda}_{0}^{y}, \hat{\lambda}_{1}^{y}, \hat{\lambda}_{0}^{x}, \hat{\lambda}_{1}^{x}$ are positive gain constants; $\tau_{1}, \tau_{2}, \omega_{1}, \psi_{1}$ denote outputs of differentiators (42)-(44) reconstructing exactly $\dot{e}(t), \ddot{e}(t), \dot{y}(t)$ and $\dot{x}(t)$, respectively, i.e., $\dot{e}(t)=\tau_{1}(t), \ddot{e}(t)=\tau_{2}(t)$ for $t \geq T_{e}^{\prime} ; \dot{y}(t)=\omega_{1}(t)$ for $t \geq T_{y}^{\prime}, \dot{x}(t)=\psi_{1}(t)$ for $t \geq T_{x}^{\prime}$, respectively; $L_{e}(t), L_{y}(t)$ and $L_{x}(t)$ stand for positive continuous functions which take the forms $L_{e}(t)=\lambda_{\max }\left(\hat{J} \hat{J}^{T}\right) \frac{c}{A}(\mathcal{Y}+$ $\left.c_{0}\right)+\mathcal{W}+\left\|\dddot{p}_{d}\right\| ; L_{y}(t)=L_{x}(t)=\lambda_{\max }\left(M^{-1}\right)\left(w^{\prime}\|v\|+w^{\prime \prime} z_{n}\left(\psi_{1}, \omega_{1}\right)^{2}+\right.$ $w^{\prime \prime \prime}+\beta^{0}$ ), where $w^{\prime}, w^{\prime \prime}$ and $w^{\prime \prime \prime}$ denote positive coefficients (constructive mobile manipulator parameters). The quantities $L_{e}(t), L_{y}(t), L_{x}(t)$ represent physically upper estimates of the norms of $\dddot{e}, \ddot{y}, \ddot{x}$ (task error jerk, manipulator joint and platform posture accelerations, respectively). Replacing $\dot{e}, \ddot{e}, \dot{y}$ and $\dot{x}$ in (29) by their corresponding estimates $\tau_{1}, \tau_{2}, \omega_{1}, \psi_{1}$ from differentiators equations (42)-(44) and using (39), we obtain the following trajectory tracking controller:

$$
\dot{v}=\hat{J}^{T} u\left(t, z_{n}\left(\psi_{1}, \omega_{1}\right), \tau_{1}, \tau_{2}\right)
$$


with

$$
u\left(t, z_{n}\left(\psi_{1}, \omega_{1}\right), \tau_{1}, \tau_{2}\right)= \begin{cases}-\frac{c}{A} \frac{s}{\|s\|}\left(\mathcal{Y}+c_{0}\right) & \text { for } s \neq 0 \\ 0 & \text { otherwise }\end{cases}
$$

which requires only measurements of $e, y$ and $x$. Based on (42)-(44), we are now in position to give the following theorem.

Theorem 2 If $e, y$ and $x$ are available from measurements and the assumptions of Theorem 1 are fulfilled then control scheme (45)-(46) guarantees stable convergence in a finite time of the task errors $(e, \dot{e}, \ddot{e})$ to the origin $(e, \dot{e}, \ddot{e})=(0,0,0)$.

Proof. The proof of Theorem 2 is a small modification of the proof of Theorem 1. Therefore, it is omitted.

In a general case, if measured task error $e=e(t)$, manipulator joint angles $y=y(t)$ and platform posture $x=x(t)$, obtained from encoders, are additionally contaminated by a measurement noise, i.e., $e(t)=e_{0}(t)+$ $\eta_{e}(t), y(t)=y_{0}(t)+\eta_{y}(t), x(t)=x_{0}(t)+\eta_{x}(t)$, where $\left\|\eta_{e}\right\|,\left\|\eta_{y}\right\|,\left\|\eta_{x}\right\| \leq$ $\rho \min \left\{L_{e}(t), L_{y}(t), L_{x}(t)\right\} ; \rho$ denotes a normalized noise magnitude (practically $\left.\rho \in\left[10^{-5}, 10^{-3}\right]\right) ; e_{0}(t), \dot{e}_{0}(t), \ddot{e}_{0}(t), y_{0}(t), \dot{y}_{0}(t), x_{0}(t)$ and $\dot{x}_{0}(t)$ stand for unknown true (noise-free) task error, task velocity error, task acceleration error, joint angles, joint velocities, platform posture and its velocity, respectively, then observers (42)-(44) should also be applied to estimate quantities $e, \dot{e}, \ddot{e}, y, \dot{y}, x$ and $\dot{x}$. Note from equations (42)-(44), that $\left\|e_{0}(t)-\tau_{0}(t)\right\| \leq$ $L_{e}(t) O(\rho) ;\left\|\dot{e}_{0}(t)-\tau_{1}(t)\right\| \leq L_{e}(t) O\left(\rho^{2 / 3}\right) ;\left\|\ddot{e}_{0}(t)-\tau_{2}(t)\right\| \leq L_{e}(t) O\left(\rho^{1 / 3}\right)$; $\left\|y_{0}(t)-\omega_{0}(t)\right\| \leq L_{y}(t) O(\rho) ;\left\|\dot{y}_{0}(t)-\omega_{1}(t)\right\| \leq L_{y}(t) O\left(\rho^{1 / 2}\right) ;\left\|x_{0}(t)-\psi_{0}(t)\right\| \leq$ $L_{x}(t) O(\rho)$ and $\left\|\dot{x}_{0}(t)-\psi_{1}(t)\right\| \leq L_{x}(t) O\left(\rho^{1 / 2}\right)$ after finite transient times [47]. Consequently, for task error norm estimates $\|\hat{e}\|=\left\|\tau_{0}\right\|,\|\dot{\hat{e}}\|=\left\|\tau_{1}\right\|$ and $\|\ddot{\hat{e}}\|=\left\|\tau_{2}\right\|$, we obtain after simple calculations the following upper estimations for controller (45)-(46) subject to measurement noise of devices: $\|\hat{e}\| \leq L_{e}(t) O(\rho) ;\|\dot{\hat{e}}\| \leq L_{e}(t) O\left(\rho^{2 / 3}\right)$ and $\|\ddot{\hat{e}}\| \leq L_{e}(t) O\left(\rho^{1 / 3}\right)$, respectively.

\section{Numerical computations}

This section demonstrates the performance of the controllers given by expressions (45)-(46) and (40)-(41) in conditions closely related to an experiment. The aim is also to show that control laws (45)-(46) and (40)-(41) are effective in counteracting the unknown both external forces exerted on the end-effector and slip reaction forces acting on the platform wheels, unknown friction forces coming from joints directly driven by the actuators as well as forces resulting from singular configurations appearing on the mechanism trajectory. The computations are carried out for the two selected mobile manipulator tasks. The

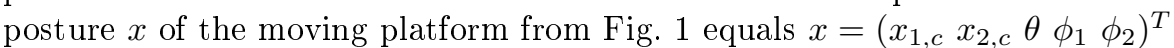
$(l=5)$ and vector $\chi$ of the lateral and longitudinal slips displacements is equal to $\chi=\left(\begin{array}{llll}\chi_{1} & \chi_{2} & \chi_{3} & \chi_{4}\end{array}\right)^{T}(k=4)$. Manipulator joint angles vector $y$ is composed of the two coordinates, i.e. $y=\left(\begin{array}{ll}y_{1} & y_{2}\end{array}\right)^{T}(n=2)$. Consequently, configuration 
$q$ whose coordinates are suitably reordered for purpose of further computa-

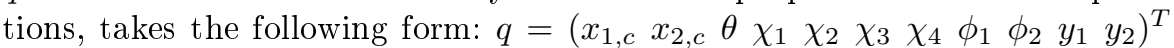
with $x_{a}=\left(\begin{array}{ll}\phi_{1} & \phi_{2}\end{array}\right)^{T}$ and $\chi_{s}=\left(\chi_{2}, \chi_{3}, \chi_{4}\right)^{T}$, respectively. Mobile manipulator from Fig. 1 operates in a two dimensional work space $(m=2)$. Hence, it becomes redundant mechanism with $l+n-k+1-m=2$ redundant degrees of freedom and $l+n-k+1=4$ actuators. In all numerical computations, SI units are used. The nominal values of both kinematic and dynamic parameters of the mobile manipulator have been taken from work [14]. The nominal values of link lengths of the holonomic manipulator are equal to $l_{1}=0.514$, $l_{2}=0.362$. The wheel radius $R$ equals $R=0.0508$. The distance between the platform wheels $2 W$ is equal to $2 W=0.364$ and $l_{a}=0.1$, respectively. The distance $d$ between the mass centre and common drive wheels axis equals $d=0$ for simplicity. The dynamic parameters take the following nominal values: wheel mass $m_{w}=0.159$; mobile platform mass $m=17.25$; masses of the first and second links of the holonomic manipulator $m_{1}=2.56$ and $m_{2}=1.07$, respectively; wheel inertia with respect to its axis $I_{w}=0.0002$; wheel inertia with respect to global $O X_{3}$ axis $I_{z}=0.0001$; platform inertia with respect to its centre of mass $I_{p}=0.297$; links inertia with respect to their centre of masses $I_{1}=0.148$ and $I_{2}=0.0228$, respectively. Let us note that two driving wheels of the mobile manipulator from Fig. 1 are rigidly connected with the platform. Hence, they do not have relative movements along lateral direction. Consequently, their lateral slips have to be equal. Thus, matrix $A$ from (2) takes in such a case the form

$$
A=\left[\begin{array}{ccccccccc}
s \theta & -c \theta & 0 & -1 & 0 & 0 & 0 & 0 & 0 \\
s \theta & -c \theta & 0 & 0 & -1 & 0 & 0 & 0 & 0 \\
c \theta & s \theta & W & 0 & 0 & -1 & 0 & -R & 0 \\
c \theta & s \theta & W & 0 & 0 & 0 & -1 & 0 & -R
\end{array}\right],
$$

where $c \theta=\cos (\theta) ; s \theta=\sin (\theta)$. The kinematic equations of the mobile manipulator from Fig. 1 take the following form:

$$
p_{e}=f_{e}(q)=\left(\begin{array}{c}
x_{1, c}+l_{a} c \theta+l_{1} c \theta 1+l_{2} c \theta 2 \\
x_{2, c}+l_{a} s \theta+l_{1} s \theta 1+l_{2} s \theta 2
\end{array}\right),
$$

where $c \theta i=\cos \left(\theta+\sum_{j=1}^{i} y_{j}\right) ; s \theta i=\sin \left(\theta+\sum_{j=1}^{i} y_{j}\right) ; i=1, \ldots, n$ and $n=2$, respectively. Due to inequality $l+n-k+1-m=2>0$, we can augment vector $p_{e}$ by additional coordinates $p_{a} \in \mathbb{R}^{2}$ of the point at which the holonomic manipulator is attached to the platform, as follows

$$
p_{a}=f_{a}(q)=\left(\begin{array}{c}
x_{1, c}+l_{a} c \theta \\
x_{2, c}+l_{a} s \theta
\end{array}\right) .
$$

The friction forces $E_{f}^{\prime}$ assumed in computations are equal to

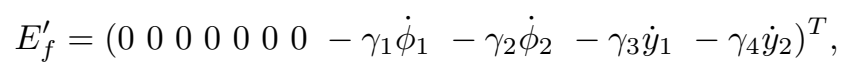


with $\gamma_{i}=0.05 ; i=1, \ldots, 4$. Matrix $D^{\prime}(q)$ from $(11)$ takes the form $D^{\prime}(q)=$ $\left(\frac{\partial f_{e}}{\partial q}\right)^{T}$. Hence, generalized force $E_{e}^{\prime}$ in (11) equals

$$
E_{e}^{\prime}=\left(\frac{\partial f_{e}}{\partial q}\right)^{T} F
$$

where $F \in \mathbb{R}^{2}$ denotes external force vector (imitating the action of e.g. a payload) exerted on the end-effector. It takes the following form (external forces of a Brownian motion type):

$$
F=\left(50+\eta_{1}(t), 20+\eta_{2}(t)\right)^{T},
$$

where $d \eta_{i}=225 \sqrt{t} X(t) d t ; X(t) \sim N(0,1) ; i=1,2 ; t \in[0,10]$. Let us note that $F$ given by (52) is globally unbounded and totally unstructured. Without loss of generality of computations, we assume that both slip ratio $s r_{j}$ and slip angle $s a_{j}, j=1,2$ are relatively small when accomplishing the robotic task. Thus, slip reaction forces (12) can be linearly approximated as

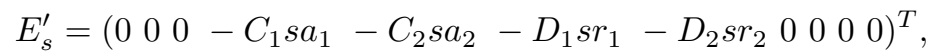

where $C_{1}=C_{2}=200 ; D_{1}=D_{2}=120$ and $\delta=10^{-5}$, respectively. Actuator matrix $B^{\prime}$ in (9) equals $B^{\prime}=\left[\begin{array}{c}0_{(7 \times 4)} \\ \mathbb{I}_{4}\end{array}\right]$ and matrices $K_{r}, K_{t}$ are assumed for simplicity to be equal to $K_{r}=K_{t}=\mathbb{I}_{4}$, respectively. The estimates for controller (45)-(46) are chosen as $A=0.5, c_{A^{\prime}}=0.01 ; \rho^{\prime}=0.4 ; \lambda_{\max }\left(J \hat{J}^{T}\right)=3.9$; $\lambda_{\max }\left(M^{-1}\right)=5.2$. In order to simplify numerical computations, rough conservative estimates of $w_{i}, i=1: 5$ have been assumed. Hence, positive constant coefficients $w_{i}$ were chosen as follows $w_{1}=1.25 ; w_{2}=0.75 ; w_{3}=0.05 ; w_{4}=0$ and $w_{5}=0.5$, respectively. The initial configuration $q(0)$ and reduced velocity

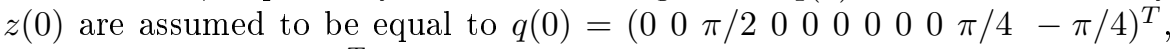
$z(0)=\left(\begin{array}{lllllll}0 & 0 & 0 & 0 & 0 & 0 & 0\end{array}\right)^{T}$, respectively. In order to accelerate the convergence process of our differentiators (42)-(44), we have chosen good initial guesses $\tau_{1}(0) ; \tau_{2}(0) ; \psi_{1}(0)$ and $\omega_{1}(0)$ in numerical computations (which imply relations $T_{e}^{\prime}, T_{x}^{\prime}, T_{y}^{\prime} \sim 0$ ) based on the nominal values of kinematic and dynamic parameters of the mobile manipulator. Hence, model-free observer (42)-(44) was run with the following initial values: $\tau_{1}(0)=\left(\begin{array}{lll}-0.2-0.15 & -0.20\end{array}\right)^{T}$; $\tau_{2}(0)=\left(\begin{array}{llll}142.16 & 0.15 & -3.06 & 1.03\end{array}\right)^{T} ; \psi_{1}(0)=\left(\begin{array}{lllll}0 & 0 & 0 & 0 & 0\end{array}\right)^{T} ; \omega_{1}(0)=\left(\begin{array}{ll}0 & 0\end{array}\right)^{T}$ and observer gains $\hat{\lambda}_{0}^{e}=1.1, \hat{\lambda}_{1}^{e}=1.4, \hat{\lambda}_{2}^{e}=2.0 ; \hat{\lambda}_{0}^{x}=\hat{\lambda}_{0}^{y}=1.1$ and $\hat{\lambda}_{1}^{x}=\hat{\lambda}_{1}^{y}=1.4$, respectively. Due to conservative nature of estimates $L_{e}(t)$, $L_{x}(t)$ and $L_{y}(t)$ in (42)-(44), they take simplified forms in our computations, as follows $L_{e}(t)=\lambda_{\max }\left(\hat{J} \hat{J}^{T}\right)\left\|u\left(t, \tau_{1}, \tau_{2}, \omega_{1}, \psi_{1}\right)\right\| \mathcal{W} ; \lambda_{\max }\left(\hat{J} \hat{J}^{T}\right)=3.9$; $L_{y}(t)=\lambda_{\max }\left(M^{-1}\right)\left(\|v\|+z_{n}^{2}\left(\psi_{1}, \omega_{1}\right)+\left\|u\left(t, \tau_{1}, \tau_{2}, \omega_{1}, \psi_{1}\right)\right\|\right)$, where $\psi_{1}=$ $\left(\psi_{1,1}, \ldots, \psi_{1,5}\right)^{T} ; \omega_{1}=\left(\omega_{1,1} \omega_{1,2}\right)^{T} ; z_{n}^{2}\left(\psi_{1} \omega_{1}\right)=c_{A^{\prime}}^{2}\left\|\psi_{1}\right\|^{2}+\left(\psi_{1,4}\right)^{2}+\left(\psi_{1,5}\right)^{2}+$ $\left\|\omega_{1}\right\|^{2}$ and $L_{x}(t)=50 L_{y}(t)$, respectively. Moreover, measured platform posture $x$, joint configuration $y$ and task error $e$ have additionally been contaminated by a measurement noise of a Brownian motion of the form $d \eta(t)=$ $\rho \sqrt{t} X(t) d t$ for $t \in[0,10] ; X(t) \sim N(0,1) ; \rho \in\left\{0,10^{-3}, 10^{-4}\right\}$, respectively. 
Different values for $\rho$ are to show the sensitivity of controller (45)-(46) on the magnitude of the amplitude of the measurement noise $\eta(t)$. In order to show also the robustness of our control schemes against singular configurations, the mobile manipulator from Fig. 1 is to accomplish two tasks.

The first task, realized by controller (45)-(46) is to track desired augmented singularity-free trajectory $p_{d}(t)$, which takes the form

$$
p_{d}(t)=(0.2 t+0.30 .5+0.25 \sin (0.2 \pi t) 0.2 t 0)^{T} .
$$

The estimate $\hat{J}(q)$ of the uncertain Jacobian matrix $J(q)$ is assumed in computations to be equal to

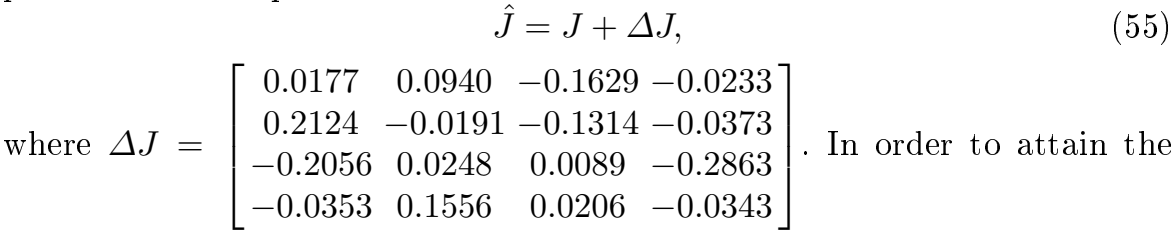

convergence of task errors $e$ at least less or equal to $10^{-4}$, the following numerical values of gain coefficients are taken for the controller: $\lambda_{0}=1 ; \lambda_{1}=31$; $\lambda_{2}=26 ; c_{0}=0.25 ; c^{\prime}=1.1 ; \beta^{0}=0.05 z_{n}\left(\psi_{1}, \omega_{1}\right)+1050$ and $\beta^{1}=0$. The results of our 'real case' computations are depicted in Figs 2-8. As is seen from Fig. 2, our controller generates tracking error estimates $\hat{e}$, which are practically for $\rho=0$ and $t \geq 4$ equal to zero compared with those generated for $\rho \in\left\{10^{-3}, 10^{-4}\right\}$. Steering signals (armature currents) $v_{1}, \ldots, v_{4}$ are depicted in Figs 3-6. As is seen from Figs 3-6, control variables $v_{1}, \ldots, v_{4}$ are absolutely continuous functions of time even for relatively large magnitude of amplitude $\rho$ of the measurement noise. As is also seen from Figs 3-6, steering signals $v_{1}, \ldots, v_{4}$ are not, in fact, sensitive to measurement noise. This is the advantage of application of the sliding techniques. Lateral and longitudinal slips of the both wheels are shown in Figs 7-8 for $\rho=10^{-3}$. As we can observe from Fig. 7, lateral slip variables $\dot{\chi}_{1}, \dot{\chi}_{2}$ take relatively large values at the beginning of the control process. This is due to the fact that initial platform orientation equals $\theta=\pi / 2$ and the platform must turn sharply by angle equal to $-\pi / 2$ to track desired trajectory. Moreover, large initial values of longitudinal slip variables $\dot{\chi}_{3}, \dot{\chi}_{4}$ in Fig. 8 are due to the fact that the platform has to accelerate in order to track desired trajectory (initial platform velocity equals zero).

The aim of the second computations is to show robustness of our transposed Jacobian control law against singular configurations. For this purpose, we assume full knowledge of the mobile manipulator kinematics and measurement availability of quantities $\dot{e}, \ddot{e}, \dot{x}$ and $\dot{y}$, respectively to show passing through singular manifold of the mechanism for control law (40)-(41). We have also assumed the numerical values of gain coefficients $\lambda_{1}, \lambda_{2}, c_{s}^{\prime}$ equal to $\lambda_{1}=11$, $\lambda_{2}=6$ and $c_{s}^{\prime}=c^{\prime}$ in this case. All other controller gains are the same as those given in the first 'real case'. In order to force singular (isolated) configurations, controller (40)-(41) has been used to track the following extended desired trajectory:

$$
p_{d}(t)=(0.2 t+0.30 .626+0.25 \sin (0.2 \pi t) 0.2 t+0.20)^{T} .
$$




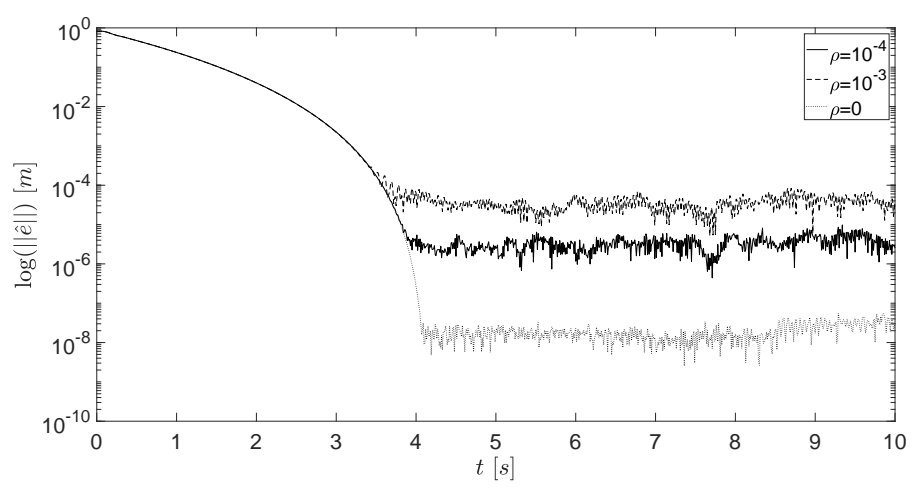

Fig. 2 Logarithm of the norm of estimated task errors $\hat{e}$ for controller (45)-(46) and singularity-free desired trajectory (54).

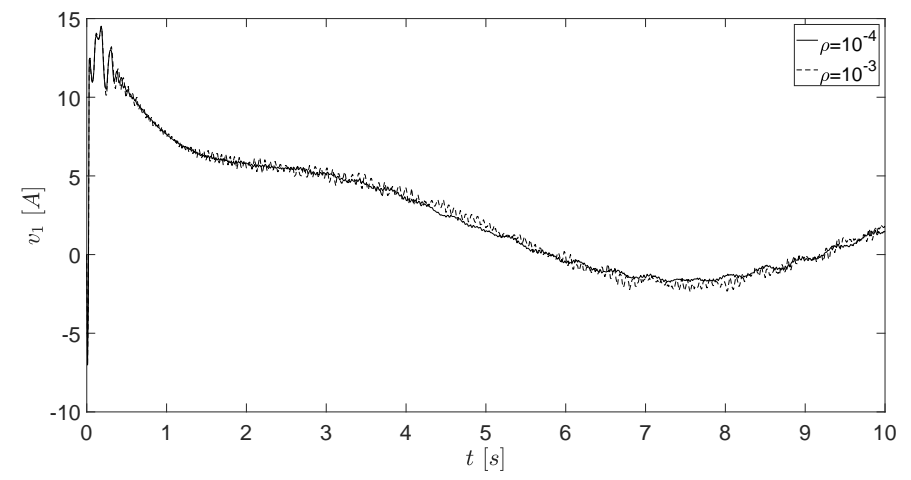

Fig. 3 Armature current $v_{1}$ for controller (45)-(46) and singularity-free desired trajectory (54).

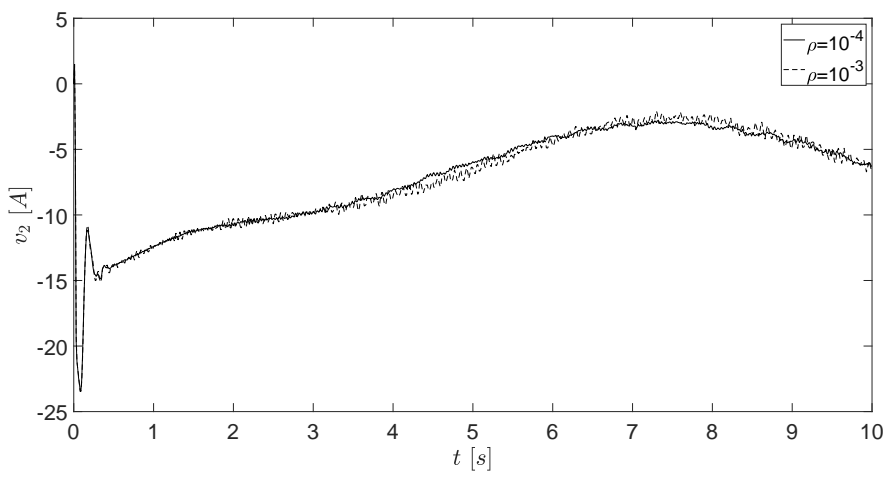

Fig. 4 Armature current $v_{2}$ for controller (45)-(46) and singularity-free desired trajectory (54). 


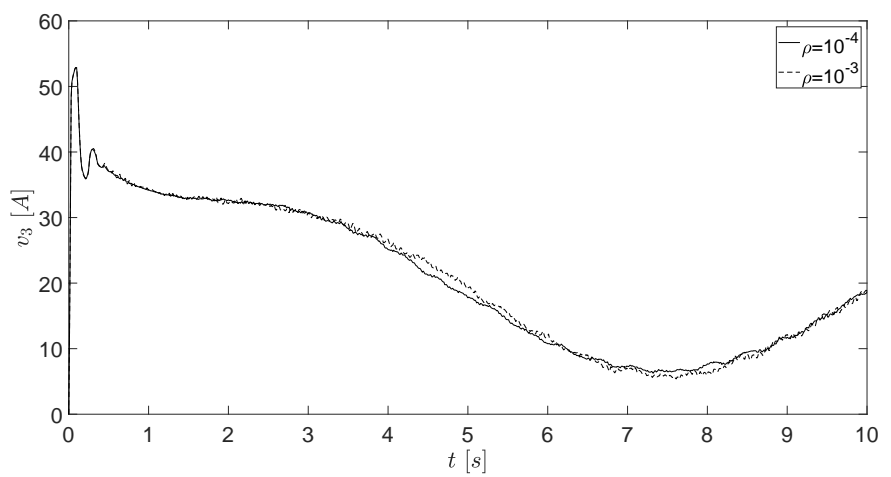

Fig. 5 Armature current $v_{3}$ for controller (45)-(46) and singularity-free desired trajectory (54).

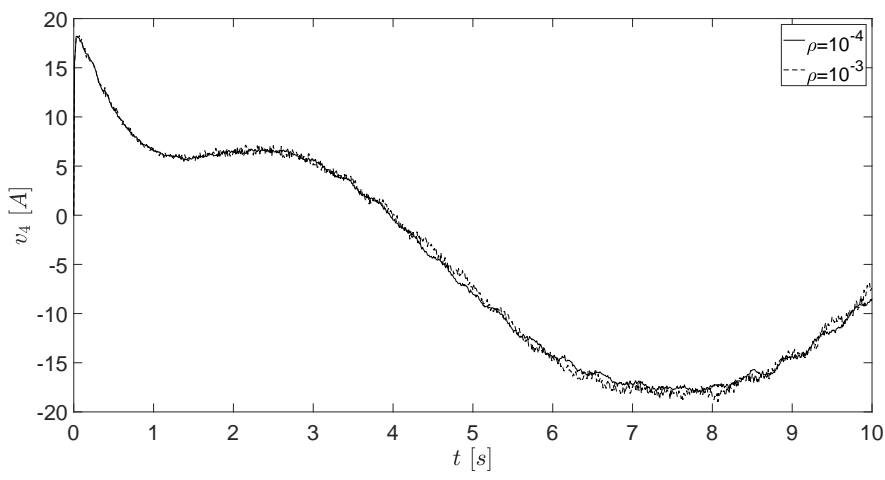

Fig. 6 Armature current $v_{4}$ for controller (45)-(46) and singularity-free desired trajectory (54).

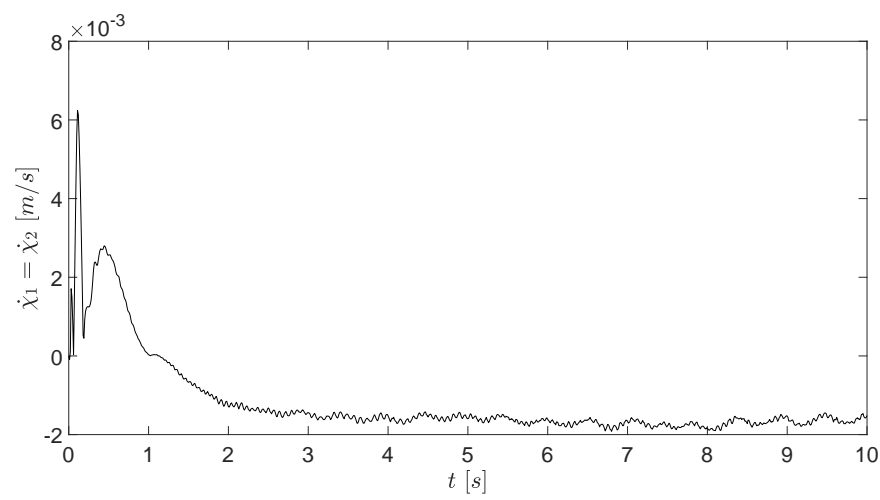

Fig. 7 Lateral slip velocities $\dot{\chi}_{1}, \dot{\chi}_{2}$ for controller (45)-(46) and singularity-free desired trajectory (54). 


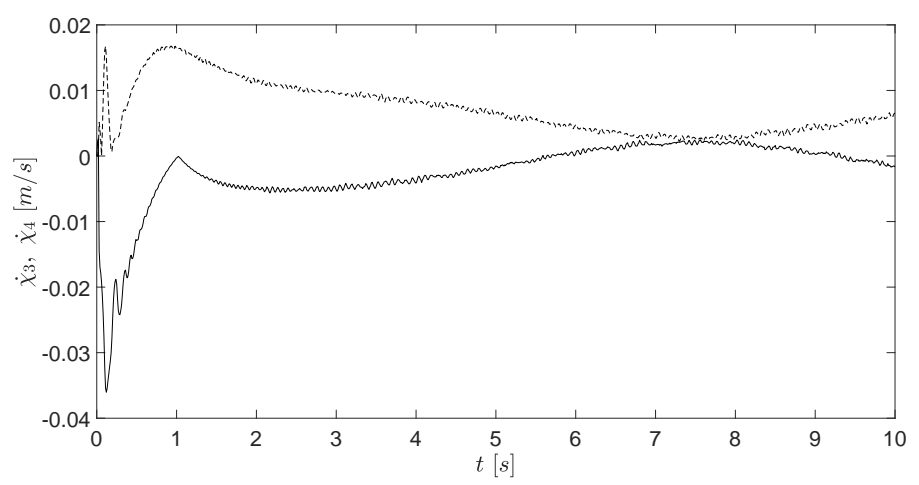

Fig. 8 Longitudinal slip velocities $\dot{\chi}_{3}$ (solid line), $\dot{\chi}_{4}$ (dashed line) for controller (45)-(46) and singularity-free desired trajectory (54).

Let us note that trajectory (56) generates infinitely many singular configurations corresponding to the set of time instances $\left\{t_{k}^{\prime}: t_{k}^{\prime}=\frac{0.5+2 k}{0.2}, k\right.$ is integer $\}$ whose Lebesgue measure equals zero provided that $e=0$. The results of the computations are depicted in Figs 9-13 which show stable finite-time convergence of the end-effector location and platform posture $p$ to desired trajectory $p_{d}$ (see Fig. 9) in the singular movement. Fig. 10 presents the passing of mobile manipulator through singular manifold $\left\{q^{\prime}: \operatorname{det}\left(J\left(q^{\prime}\right)\right)=0\right\}$ in neighbourhoods of time instances $t^{\prime} \in\{2.5,12.5\}$. Such a passing may be treated as instantaneous acting a disturbance signal on the mobile manipulator. Visible variations of $\|e\|$ and $v_{1}, v_{2}, v_{3}, v_{4}$ (Figs 9,11) in small neighbourhoods of $t^{\prime} \in\{2.5,12.5\}$ are a consequence of violating the assumption (21) in a set of time instances of non-zero Lebesgue measure by the corresponding numerical value of $\lambda_{\min }\left(J\left(q^{\prime}\right) J^{T}\left(q^{\prime}\right)\right)$ and compensating by controller (40)-(41) the reaction force $\left.\left(\frac{\partial f_{e}}{\partial q}\right)^{T} F_{g}\right|_{q=q\left(t_{i}^{\prime}\right)}$ (manipulator velocity $z$ tends to approach large values in neighbourhoods of $t_{1}^{\prime}=2.5$ and $t_{2}^{\prime}=12.5$, respectively). These variations may be made arbitrarily small by choosing a suitably large value for $c_{s}^{\prime}$. Figs 12-13 present lateral and longitudinal slip velocities. Let us observe that in neighbourhoods of time instances $t^{\prime} \in\{2.5,12.5\}$, longitudinal slip velocities take relatively large values. This is a consequence of large platform posture and manipulator joint velocities occurring in neighbourhoods of singular configurations.

\section{Conclusions}

A new class of task space TSM controllers with finite-time stability when tracking a desired end-effector trajectory by the mobile manipulator has been proposed in this paper. On account of the fact that external forces acting on the mechanism are unknown and some configuration variables (slip velocities) 


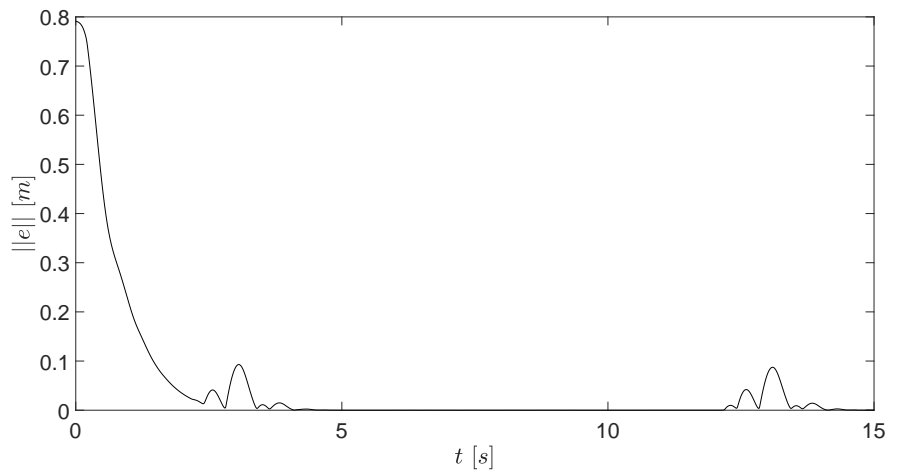

Fig. 9 Norm of task errors $e$ for controller (40)-(41) and singular desired trajectory (56).

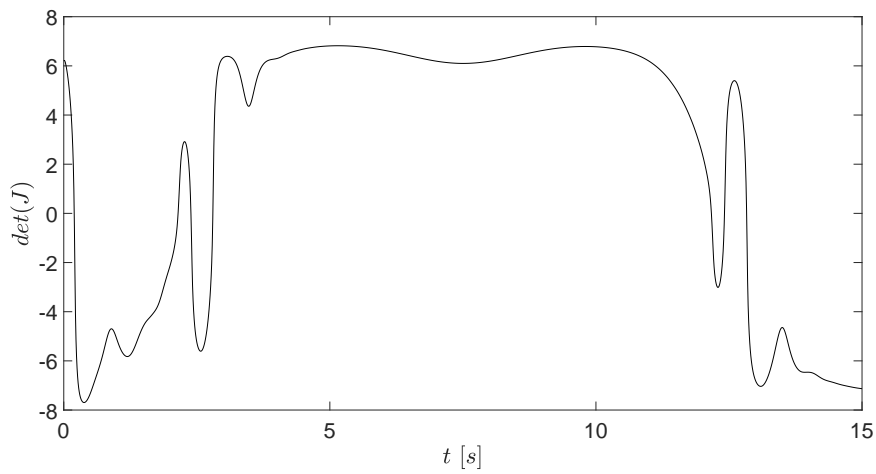

Fig. 10 Determinant of extended Jacobian matrix $J(q)$ for controller (40)-(41) and singular desired trajectory $(56)$

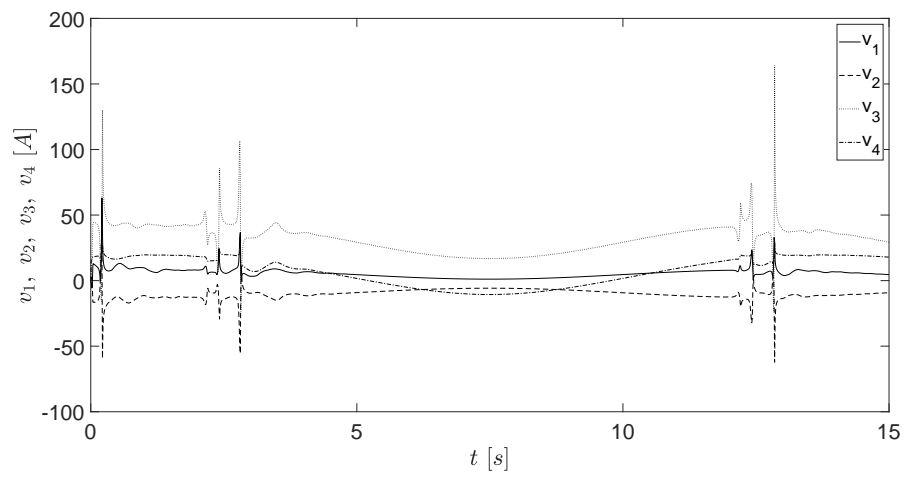

Fig. 11 Armature currents $v_{1}, v_{2}, v_{3}, v_{4}$ for controller (40)-(41) and singular desired trajectory (56). 


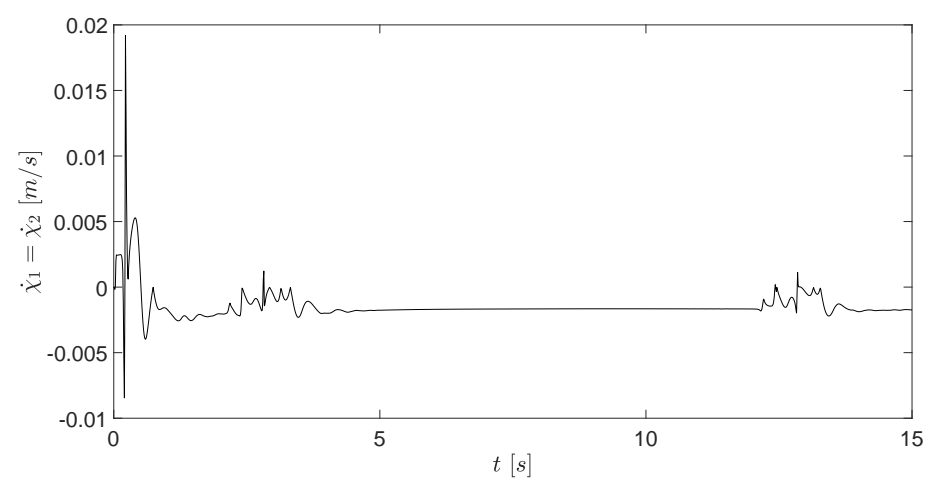

Fig. 12 Lateral slip velocities $\dot{\chi}_{1}, \dot{\chi}_{2}$ for controller (40)-(41) and singular desired trajectory (56).

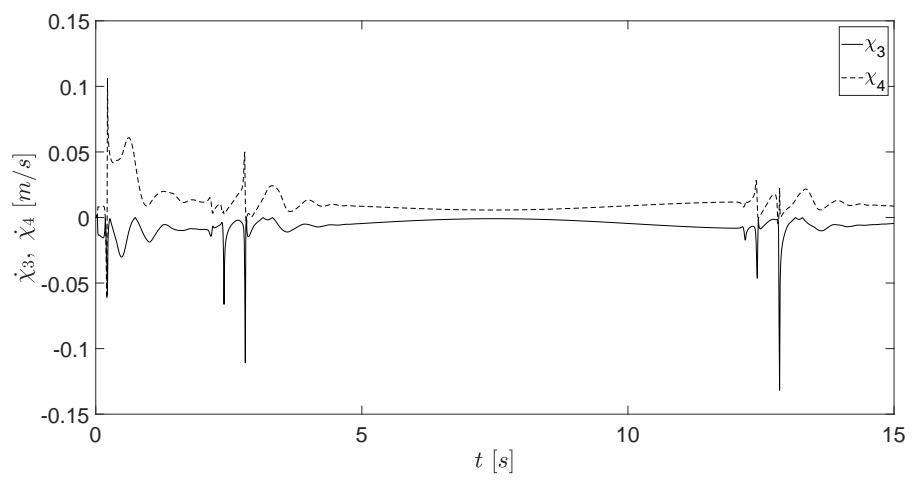

Fig. 13 Longitudinal slip velocities $\dot{\chi}_{3}$ (solid line), $\dot{\chi}_{4}$ (dashed line) for controller (40)-(41) and singular desired trajectory $(56)$.

are also hard to sense, we have offered a sliding technique of the second order, which seems to be both effective in counteracting those undesirable forces and does not need force sensors nor slip variables to track desired trajectory. Moreover, our controllers provide physically realizable steering signals which are absolutely continuous functions of time. Another feature of the control laws proposed is the elimination of the Jacobian matrix inverse (or pseudoinverse) from the trajectory tracking. Instead, estimated Jacobian transpose matrix has been used. Applying the Lyapunov stability theory, control strategies (45)-(46) are shown to be finite-time stable by fulfilment of practically reasonable assumptions. Furthermore, finite-time stability proof with transpose Jacobian control law (45)-(46) seems also to be a theoretically novel result in the problem of tracking the desired trajectory. Numerical computations have shown that controllers (45)-(46) and (40)-(41) well perform under conditions of both unknown external forces and measurement noise of the mechanism 
encoders. Although our transposed Jacobian controllers need some knowledge extracted from both the system kinematics and dynamics of the mechanism, the approach is able to handle uncertainties in kinematics and dynamics of the mobile manipulator as well as unknown external forces.

\section{A summary statement}

Conflict of Interest: The authors declare that they have no conflict of interest.

\section{References}

1. Rani, M., Kumar, N., Singh, H., P.: Efficient position/force control of constrained mobile manipulators. International Journal of Dynamics and Control, 6, 1629-1638 (2018)

2. Dong, W.: On trajectory and force tracking control of constrained mobile manipulators with parameter uncertainty. Automatica, 38(9), 1475-1484 (2002)

3. Baspinar, C.: On robust position/force control of robot manipulators with constraint uncertainties. Proc. of the 10th IFAC Symposium on Robot Control, 555-560 (2012)

4. Li, Z., Ge, S.S.: Fundamentals of modeling and control of mobile manipulators. $C R C$ (2013)

5. Li, Z., Ge, S.S., Adams, M., Wijesoma, W.S.: Robust adaptive control of uncertain constrained nonholonomic mobile manipulators. Automatica, 44, 776-784 (2008)

6. Kaczmarek, M., Domski, W., Mazur, A.: Position-force control of mobile manipulatornonadaptive and adaptive case. Archives of Control Sciences, 27(4), 487-503 (2017)

7. Balleieul, J.: Kinematic programming alternatives for redundant manipulators. In Proc. IEEE Int. Conf. on Robotics and Automation. 2, 722-728, (1985)

8. White, G.D., Bhatt, R.M., Tang, C.P., Krovi, V.N.: Experimental evaluation of dynamic redundancy resolution in a nonholonomic wheeled mobile manipulator. IEEE/ASME Trans. Mechatron. 14(3), 349-357 (2009)

9. White, G.D., Bhatt, R.M., Krovi, V.N.: Dynamic redundancy resolution in a nonholonomic wheeled mobile manipulator. Robotica, 25(2), 147-156 (2007)

10. Nemec, B., Zlaypah, L.: Force control of redundant robots in unstructured environment. IEEE Trans. Industrial Electronics. 49(1), 233-240 (2002)

11. Zhang, Y., Yan, X., Chen, W.: QP-based refined manipulability-maximizing scheme for coordinated motion planning and control of physically constrained wheeled mobile redundant manipulators. Nonlinear Dyn. 85, 245-261, (2016)

12. Zhong G., Kobayashi, Y., Hoshino, Y., Emaru, T.: System modeling and tracking control of mobile manipulator subject to dynamic interaction and uncertainty. Nonlinear Dyn. $73,167-182(2013)$

13. Inoue, F., Murakami, T., Ohnishi, K.: A motion control of mobile manipulator with external force. IEEE/ASME Trans. Mechatron. 6(2), 137-142 (2001)

14. Boukattaya, M., Mezghani, N., Damak, T.: Adaptive motion/force control of uncertain nonholonomic mobile manipulator with estimation of unknown external force. Multibody System Dynamics. 44, 223-250 (2018)

15. Dehghan, S., Danesh, M., Sheikholeslam, F.: Adaptive hybrid force/position of robot manipulators using an adaptive force estimator in the presence of parametric uncertainty. Adv. Robot. 29(4), 209-223 (2015)

16. Boukattaya, M., Jallouli, M., Damak, T.: On trajectory tracking control for nonholonomic mobile manipulators with dynamic uncertainties and external torque disturbances. Robot. Auton. Syst. 60(12), 1640-1647 (2012)

17. Gierlak, P., Szuster, M.: Adaptive position/force control for robot manipulator in contact with a flexible environment. Robotics and Autonomous Systems. 95, 80-101 (2017)

18. Motte, I., Campion, G.: A Slow Manifold Approach for the Control of Mobile Robots Not Satisfying the Kinematic Constraints. IEEE Trans. Robotics and Automation, 16(6), $875-880(2000)$. 
19. Novel, A., B., Campion, G. Bastin G.: Control of Wheeled Mobile Robots Not Satisfying Ideal Velocity Constraints: A Singular Perturbation Approach. Int. J. Robust and Nonlinear Control, 5, 243-267, (1995).

20. Ploeg, J., Schouten, H., E., Nijmeijer, H.: Position Control of a Wheeled Mobile Robot Including Tire Behaviour. IEEE Trans. Intelligent Transp. Systems, 10(3), 523-533, (2009).

21. Tian, Y., Sidek, S., N., Sarkar, N.: Tracking Control for Nonholonomic Wheeled Mobile Robot with Wheel Slip Dynamics, in Proc. ASME 2009 Dynamic Systems and Control Conference DSCC2009, (2009).

22. Sidek, N., Sarkar, N.: Exploiting wheel slips of mobile robots to improve navigation performance. Advanced Robotics, 27(8), 627-639, (2013).

23. Ward, C., Iagnemma, K.: A Dynamic-Model-Based Wheel Slip Detector for Mobile Robots on Outdoor Terrain. IEEE Trans. Robotics, 24(4), 821-821, (2008).

24. Chen, M.: Disturbance Attenuation Tracking Control for Wheeled Robots With Skidding and Slipping. IEEE Trans. Industrial Electronics, (2016).

25. Wang., Y., Jia, Y., Li, X., Xi, N.: Dynamics Modelling of A Mobile Manipulator for Wheel Slip Avoidance. in Proc. the 2011 IEEE Intern. Conf. Robotics and Biomimetics, (2011).

26. Song, T., Xi, F., Guo, S., Tu, X., Li, X.: Slip Analysis for a Wheeled Mobile Manipulator. J. Dynamic Systems, Measurement and Control, 140, (2018).

27. Edwards, C., Spurgeon., S., K.:Sliding mode control: Theory and Application, Taylor and Francis: London, (1998).

28. Utkin, V., I.: Sliding modes in Optimization and Control Problems, Springer: New York, (1992).

29. Fridman, L.: Singularity perturbed analysis of chattering in relay control systems, IEEE Trans. Automatic Control, 47(12), 2079-2084, (2002).

30. Seraji, H., Colbaugh, R.: Improved configuration control for redundant robots. J. Robot Syst. 6, 897-928 (1990)

31. Galicki, M. "Tracking the kinematically optimal trajectories by mobile manipulators", Journal of Intelligent \& Robotic Systems 93(3-4), 635-648 (2019).

32. Wang, J.: Manipulation of a mobile modular manipulator interacting with the environment with the assistance of tactile sensing feedback. Int. J. Humanoid Robot. 8(4), 777-793 (2011)

33. Phong, D., Choi, J., Lee, W., Kang, S.: A novel method for estimating external force: simulation study with a 4-DOF robot manipulator. Int. J. Precis. Eng. Manuf. 16(4), 755-766 (2015)

34. Campion, G., Bastin, G., Andrea-Novel B. D.: Structural properties and classification of kinematic and dynamic models of wheeled mobile robots. IEEE Trans. Robot. Autom. 12(1), 47-62 (1996)

35. Seraji, H.: A unified approach to motion control of mobile manipulators. Int. J. Robot. Res. 17(2), 107-118 (1998)

36. Galicki, M.: Inverse kinematics solution to mobile manipulators. Int. J. Robotics Res. 22(12), 1041-1064 (2003)

37. Filippov, A., F.: Differential Equations with Discontinuous Right-hand Side. Kluwer. Dordrecht, Netherlands, (1988)

38. Cheah CC., Lee, K., Kawamura, S., Arimoto, S. (2000) Asymptotic stability control with approximate Jacobian matrix and its application to visual servoing. In Proc. IEEE Decision and Control, New York, NY, USA, pp. 3939-3944.

39. Cheah, C., C.: On duality of inverse Jacobian and transpose Jacobian in task-space regulation of robots. In Proc. IEEE Int. Conf. on Robotics and Automation. 2571-2576 (2006)

40. Moosavian, S., A., A., Papadopoulos, E.: Modified transpose Jacobian control of robotic systems. Automatica. 1226-1233 (2007)

41. Defoort, M., Floquet, T., Kokosy, A., Perruquetti, W. (2009). A novel higher order sliding mode control scheme. Syst. Control Lett. 58, pp. 102-108

42. Defoort, M., Floquet, T., Kokosy, A., Perruquetti, W. (2017). Higher order sliding modes in collaborative robotics. In: Lecture Notes in Control and Information Sciences Book Series (LNCIS), 412, pp. 409-437. 
43. Galicki, M. (2016) Finite-time trajectory tracking control in a task space of robotic manipulators, Automatica, vol. 67, pp. 165-170.

44. Galicki, M., (2015) Finite-time control of robotic manipulators, Automatica, 51, pp. 49-54.

45. Atasi, A., N., and Khalil, H., K. (2000). Separation results for the stabilization of nonlinear systems using different high-gain observer designs, Syst Control Lett, 39, 183-191.

46. Levant, A., Livne, M. (2012). Exact differentiation of signals with unbounded higher derivatives. IEEE Transactions on Automatic Control, 57(4), 1076-1080.

47. Levant, A. (2003). Higher-order sliding modes, differentiation and output-feedback control, Intern. J. of Control, 76(9-10), 924-941.

48. Politiz, Z., Smith, P.: Classification of textured surfaces for robot navigation using continuous transmission frequency-modulated sonar signatures. Int. J. Rob. Res. 20, $107-128,(2001)$ 